Research Article

\title{
Time-Dependent Lagrangian Energy Systems on Supermanifolds with Graph Bundles
}

\author{
Cansel Aycan (iD) and Simge Şimşek (D) \\ Science Faculty, Department of Mathematics, Pamukkale University, 20020 Denizli, Turkey \\ Correspondence should be addressed to Cansel Aycan; aycancansel17@gmail.com
}

Received 9 January 2021; Revised 16 March 2021; Accepted 11 April 2021; Published 28 April 2021

Academic Editor: Ismail Naci Cangul

Copyright (c) 2021 Cansel Aycan and Simge Şimşek. This is an open access article distributed under the Creative Commons Attribution License, which permits unrestricted use, distribution, and reproduction in any medium, provided the original work is properly cited.

\begin{abstract}
The aim of this article is firstly to improve time-dependent Lagrangian energy equations using the super jet bundles on supermanifolds. Later, we adapted this study to the graph bundle. Thus, we created a graph bundle by examining the graph manifold structure in superspace. The geometric structures obtained for the mechanical energy system with superbundle coordinates were reexamined with the graph bundle coordinates. Thus, we were able to calculate the energy that occurs during the motion of a particle when we examine this motion with graph points. The supercoordinates on the superbundle structure of supermanifolds have been given for body and soul and also even and odd dimensions. We have given the geometric interpretation of this property in coordinates for the movement on graph points. Lagrangian energy equations have been applied to the presented example, and the advantage of examining the movement with graph points was presented. In this article, we will use the graph theory to determine the optimal motion, velocity, and energy of the particle, due to graph points. This study showed a physical application and interpretation of supervelocity and supertime dimensions in super-Lagrangian energy equations utilizing graph theory.
\end{abstract}

\section{Introduction}

Many of the problems based on Lagrangian energy equations in classical mechanics may be solved using Euclidian space. It is well known that modern differential geometry has an important role too. Many of these problems have not been calculated in superspace before. One reason may be the difficulty that the metric structure of superspace differs from Euclidean space. Using jet bundles and working with bundle coordinates is one of the most convenient ways to obtain the time-dependent Lagrangian energy equations. Therefore, in this study, supercoordinate structure will be formed on jet bundles and superenergy equations will be obtained by using this.

Mathematicians, working in superspace, assume that there is a natural phenomenon with supergeometry and supersymmetry for explaining physical phenomena occurring in even- and odd-dimensional Euclid space. Thus, we prefer to solve Lagrangian energy equations on the superspace, which is based on a jet bundle structure. Inclusion of time dimension for solving Lagrangian energy equations on superspace is an important parameter that improves the super-Lagrangian system for which we propose to take time derivative coordinates on the jet bundle.

On the other hand, graph theory is the most suitable method for planning a movement that completes a path as soon as possible. In this study, we will compare the graph bundle structure and the bundle structure that we use in calculating energy equations in the mechanical system. We will combine these two bundle structures and adapt them in the superspace. We will be able to define the motion depending on time with graph points and also form a graph vector field with graph vectors. Thus, we will combine the geometric concepts used in calculating Lagrangian energy equations with graph theory, and we will calculate the energy equation with a completely new approach. A study has been done on the graphs of the logarithmic spiral curve in [1]. In [2], using the graph theory, the study of Euler-Lagrange 
energy structure in neural networks has been examined. Cangül et al. [3, 4] is a basic resource on graph theory. Graph manifold structure is defined in [5-7]. Topological properties of the graph manifold are investigated in [8]. Graph bundle structure is examined in [9].

Aycan [10] proved the Lagrange energy equations on the jet bundle structure containing the time dimension. Also, in [11], Lagrangian energy equations are developed by forming jet bundles on a complex space. Lagrangian equations are solved with real bundles by [12-14]. Mechanical systems with time parameter were investigated in $[15,17]$. The other studies [18-24] showed the fundamental of supergeometric structures. But none of them could be solved with superjet bundles that include the time dimension in the superspace. Thus, the main contribution of this study is to obtain superLagrange energy equations with bundle structures structured with body and soul and also even and odd dimensions, and we will form it with a graph bundle with time dimension. The advantage of this solution method is that the energy equations found in superspace can be projected or comparable with energy equations in real space.

In previous studies, super-Lagrangian equations in supermanifolds have not been studied for superderivative coordinates. There for this study improves this equation in superderivative coordinates with body and soul and also even and odd dimensions.

A brief introduction of Lagrangian systems is given in the following way.

If $M$ is an $m$-dimensional configuration manifold and $L: \mathrm{TM} \longrightarrow R$ is a regular Lagrangian function, then there is a unique vector field $\varepsilon_{L}$ on TM and $\Omega_{L}$ is a 2 -form on TM, such that

$$
i_{\varepsilon_{L}} \Omega_{L}=d_{E_{L}}
$$

where $E_{L}$ is Lagrangian energy associated with $L[12,15]$. The Euler-Lagrange vector field $\varepsilon_{L}$ is a semispray or second-order differential equation on $M$ since its integral curves are the solutions of the Euler-Lagrange equations $[12,15]$. The triple (TM, $\left.\Omega_{L}, L\right)$ is called the Lagrangian system on the tangent bundle TM [12]. The coordinate system on TM is $\left(q_{i}, v_{i}\right)$. For created to time-dependent Lagrange systems are used jet bundles. When studying energy systems, it will be very convenient for obtaining energy equations with the jet bundle structure because the motion depends on time and therefore to take the time as a coordinate. At the same time, the jet bundle $J(R, M)$ is the velocity space of the manifold $M . J(R, M)$ is isomorphic to $R \times \mathrm{TM}$. The coordinate system on $J(R, M)$ is $\left(t, q_{i}, v_{i}\right)$. On jet bundles, then there is a unique vector field (or semispray) $\varepsilon_{L}$ and a 2 -form called as Poincaré-Cartan 2-form $\Omega_{L}$ again so that they provide the following equations:

$$
\begin{gathered}
i_{\varepsilon_{L}} \Omega_{L}=0, \\
i_{\varepsilon_{L}} \mathrm{~d} t=1 .
\end{gathered}
$$

Here, $L: R \times \mathrm{TM}=J(R, M) \longrightarrow R$ is the Lagrangian function. The triple $\left(J(R, M), \Omega_{L}, \varepsilon_{L}\right)$ is called the timedependent Lagrangian system. The Euler-Lagrange vector field $\varepsilon_{L}$ is locally expressed as

$$
\begin{aligned}
\varepsilon_{L} & =\frac{\partial}{\partial t}+v_{i} \frac{\partial}{\partial q_{i}}+\varepsilon_{i} \frac{\partial}{\partial v_{i}} \\
\varepsilon_{i} & =\varepsilon_{i}\left(t, q_{i}, v_{i}\right) .
\end{aligned}
$$

We have to define Poincaré-Cartan 1-form to get Poincaré-Cartan 2-form. The Poincaré-Cartan 1-form on $J(R, M)$ associated with $L$ is

$$
\alpha_{L}=d_{J} L+L \mathrm{~d} t
$$

The Poincaré-Cartan 2-form associated with $L$ is

$$
\Omega_{L}=\mathrm{d} d_{J_{L}}+\mathrm{d} L \wedge \mathrm{d} t
$$

When solving equation (2),

$$
\frac{\mathrm{d}}{\mathrm{d} t}\left(\frac{\partial L}{\partial v_{i}}\right)-\frac{\partial L}{\partial q_{i}}=0,
$$

is obtained. Equation (2) is called as time-dependent Euler-Lagrange energy equation. The same equation would be found if the solution is made in equation (1) because the Lagrange energy equation is similar. When we work in different spaces in our previous work, we have seen that the obtained Lagrange energy equation can be reduced to (6). In this study, we will obtain the energy equation by creating the necessary geometric structures to obtain the Lagrange energy equation in superspace and compare it with equation (6).

\section{Bundles on Superspace}

In this section, we will define a bundle in superspace by using the bundle definitions in real space and graph theory. Then, we will form the geometric structures required for the solution of the mechanical system firstly with the jet bundle coordinates and then graph bundle coordinates. With the solution of this mechanical system, we will obtain the Lagrangian energy equation.

Definition 1. Let $(E, \pi, M)$ be a bundle. Here, $E$ is called the total space, $\pi$ is the projection, and $M$ is the base space. This bundle is denoted by $\pi$ or $E$. For each point $p \in M$, the subset $\pi^{-1}(p)$ of $E$ is called as fiber over $p$. The set of fibers is shown as $E_{p}$. So the bundle $(E, \pi, M)$ can also be called a fibrous manifold. $F$ is the typical fiber of the bundle, and for the map $t: E \longrightarrow M \times F,(F, t)$ is called a trivialization.

The first jet manifold of $\pi$ is the set $\left\{j_{p}^{1} \varnothing: p \in M, \varnothing \in \Gamma_{p}(\pi)\right\}$ and denoted by $J^{1} E$ or $J^{1} \pi$. Here, $\varnothing: M \longrightarrow E$ is a section of $\pi$. If it is satisfying the condition $\pi \circ \varnothing=i d_{M}$, then the set of all sections of $\pi$ will be denoted by $\Gamma(\pi) . \phi, \psi \in \Gamma_{p}(\pi)$, if $\phi(p)=\psi(p)$ and $\left.\left(\partial \phi^{\alpha} / \partial x_{i}\right)\right|_{p}=$ $\left.\left(\partial \psi^{\alpha} / \partial x_{i}\right)\right|_{p}$ and this is an equivalence relation and the equivalence classes containing $\varnothing$ are called the first jet at the $p$-point. These are denoted by $j_{p}^{1} \varnothing$. Also, the triple $\left(J^{1} E, \pi_{1}, M\right)$ is the first jet bundle and denoted by $J^{1} E$. Here, $\pi_{1}: J^{1} E \longrightarrow M$ is a surjective submersion. TM is the tangent bundle of the manifold $M$. (TM, $\left.p_{r_{1}}, M\right)$ is named as tangent bundle. At the same time, $J^{1} E$ is isomorphic to TM, $J^{1} E \cong \mathrm{TM}$, that is, the first jet is also a tangent vector [10]. 
Let $(U, u)$ be an adapted coordinate system on $E$, where $u=\left(x_{i}, u^{\alpha}\right)$. The induced coordinate system on $J^{1} E$ is denoted by $\left(x_{i}, u_{\alpha}, u_{\alpha}^{i}\right)$, where $x_{i}\left(j_{p}^{1} \varnothing\right)=x_{i}(p), u_{\alpha}\left(j_{p}^{1} \varnothing\right)=$ $u^{\alpha}(\varnothing(p))$, and new functions $u_{\alpha}^{i}\left(j_{p}^{1} \varnothing\right)=\left(\partial u^{\alpha} / \partial x_{i}\right)(p)$ are known as derivative coordinates.

We will define the superbundle with these properties.

Definition 2. A superbundle is a triple $\left(E^{*}, \pi^{*}, M^{*}\right)$ where $E^{*}$ and $M^{*}$ are supermanifolds and $\pi^{*}: E^{*} \longrightarrow M^{*}$ is a surjective submersion. This bundle is denoted by $\pi^{*}$ or $E^{*}$. The first superjet manifold of $\pi^{*}$ is denoted by $J^{1} \pi^{*}$ or $J^{1} E^{*}$. It is the set $\left\{j_{p}^{1} \varnothing: p \in M^{*}, \varnothing \in \Gamma_{p}\left(\pi^{*}\right)\right\}$. Here, $\varnothing$ is a map, and $\varnothing: M^{*} \stackrel{\longrightarrow}{\longrightarrow} E^{*}$ is called as supersection of $\pi^{*}$. If it satisfies the condition $\pi^{*} \circ \varnothing=\mathrm{id}_{M^{*}}$, then the set of all supersections of $\pi^{*}$ will be denoted by $\Gamma\left(\pi^{*}\right)$. The equivalence classes containing $\varnothing$ are called the first jet at the $p$ point and denoted by $j_{p}^{1} \varnothing$. Also, $\phi, \psi \in \Gamma_{p}(\pi)$, if $\phi(p)=$ $\psi(p)$ and $\left.\left(\partial \phi^{\alpha} / \partial x_{i}\right)\right|_{p}=\left.\left(\partial \psi^{\alpha} / \partial x_{i}\right)\right|_{p}$, and this is an equivalence relation and the equivalence classes containing $\varnothing$ are called the first jet at the $p$-point. Then, the first superjet bundle is the triple $\left(J^{1} E^{*}, \pi_{1}, M^{*}\right)$ and denoted by $J^{1} E^{*}$. $J^{1} E^{*}$ is isomorphic to $\mathrm{TM}^{*}, J^{1} E^{*} \cong \mathrm{TM}^{*}$, that is, the first superjet is also a supertangent vector.

The induced coordinate system on $J^{1} E^{*}$ will be obtained as follows.

In this paper, we accept the manifold $E^{*}$ as a supermanifold with $m+n$ dimension, and the manifold $M^{*}$ is a supermanifold with $m$-dimension. Let us show the adapted coordinate system on $M^{*}$ is $\left(x_{i}\right)$. Since this is a superspace coordinate, its open representation will be made as follows:

$$
x_{i}=x_{i_{b}}+x_{i_{s}}=x_{i_{b}}+x_{i_{s_{e}}}+x_{i_{s_{o}}}, \quad(1 \leq i \leq m),
$$

where $x_{i_{b}}$ is the body part of $x_{i}$ and $x_{i_{s}}$ is the soul part of $x_{i}$. On the other hand, $x_{i_{s}}$ has even and odd parity. We will show the even parity of $x_{i_{s}}$ as $x_{i_{s}}$ and the odd parity of $x_{i_{s}}$ as $x_{i_{s}}$. We show the adapted coordinate system on $E^{*}$ is $\left(x_{i}, u^{\alpha}\right)$. Similarly, the open representation of $u^{\alpha}$ is taken as follows:

$$
u^{\alpha}=u^{\alpha_{b}}+u^{\alpha_{s}}=u^{\alpha_{b}}+u^{\alpha_{s_{e}}}+u^{\alpha_{s_{o}}}, \quad(1 \leq \alpha \leq m+n),
$$

where $u^{\alpha_{b}}$ is the body part of $u^{\alpha}$ and $u^{\alpha_{s}}$ is the soul part of $u^{\alpha}$. On the other hand, $u^{\alpha}$ has also even and odd parity. We will show the even parity of $u^{\alpha_{s}}$ as $u^{\alpha_{s_{e}}}$ and the odd parity of $u^{\alpha_{s}}$ as $u^{\alpha_{s_{o}}}$. For simplicity, the body indexes symbolize as $b$ and the soul indexes symbolize as $s$. Also, we will obtain the derivative coordinates of superjet bundle $J^{1} \pi^{*}$ as follows. The important point to be considered here is that the derivatives of the soul and body parts must be taken separately within themselves. We saw that the results were very different from what was desired in all the examinations we made by using the mixed derivatives for body and soul parts. So, the coordinates of $J^{1} \pi^{*}$ are shown as follows:

$$
\left(x_{i}, u_{\alpha}, u_{\alpha}^{i}\right)=\left(x_{i_{b}}, x_{i_{s}}, u^{\alpha_{b}}, u^{\alpha_{s}}, u_{i ; b, s}^{\alpha ; b, s}\right) .
$$

Here, we denote the derivative coordinates $u_{i ; b, s}^{\alpha ; b, s}$ distinctly as follows: $u_{i ; b}^{\alpha ; b}=\left(\partial u^{\alpha b} / \partial x_{i b}\right) \quad$ (derivative of body coordinates according to body coordinates).

$u_{i ; s}^{\alpha ; s}=\left(\partial u^{\alpha s} / \partial x_{i s}\right)$ (derivative of soul coordinates according to soul coordinates).

Here, $u_{i ; s}^{\alpha ; s}$ has also even and odd parity too. We will show the even parity of $u_{i ; s}^{\alpha ; s}$ as $u_{i ; s_{e}}^{\alpha ; s_{e}}$ and the odd parity of $u_{i ; s}^{\alpha ; s}$ as $u_{i ; s_{o}}^{\alpha ; s_{o}}$.

Also,

$u_{i ; s_{e}}^{\alpha ; s_{e}}=\left(\partial u^{\alpha s_{e}} / \partial x_{i s_{e}}\right)$ (derivative of even soul coordinates according to even soul coordinates).

$u_{i ; s_{o}}^{\alpha ; s_{o}}=\left(\partial u^{\alpha s_{o}} / \partial x_{i s_{o}}\right)($ derivative of odd soul coordinates according to odd soul coordinates).

If these more explicit representations of derivative coordinates are used, we can write the adapted coordinate system of superjet bundle as

$$
\left(x_{i_{b}}, x_{i_{s_{e}}}, x_{i_{s_{o}}}, u^{\alpha_{b}}, u^{\alpha_{s_{e}}}, u^{\alpha_{s_{o}}}, u_{i ; b}^{\alpha ; b}, u_{i ; s_{e}}^{\alpha ; s_{e}}, u_{i ; s_{o}}^{\alpha ; s_{o}}\right) .
$$

If we want to create a time-dependent jet bundle, we must take the real space as the base space. So, the triple we will use is $\left(J^{1} E, \pi, R\right)$ and its coordinated system is $\left(t, x_{i}, \dot{x}_{i}\right)$. The adapted coordinate system on $E$ is $\left(x_{i}\right)$, on $R$ is $(t)$, and also on $J^{1} E$ is $\left(\dot{x}_{i}\right)$ :

$$
\dot{x}_{i}=\frac{\mathrm{d} x_{i}}{\mathrm{~d} t} .
$$

Accordingly, time-dependent jet bundle structure can also be formed in superspace. The supertime-dependent jet bundle structure will be shown as $\left(J^{1} E^{*}, \pi^{*}, R^{*}\right)$. So, the coordinates of this bundle are shown as follows:

$$
\left(t, x_{i}, \dot{x}_{i}\right)=\left(t_{b}, t_{s_{e}}, t_{s_{o}}, x_{i_{b}}, x_{i_{s_{e}}}, x_{i_{s_{o}}}, \dot{x}_{i_{b}}, \dot{x}_{i_{s_{e}}}, \dot{x}_{i_{s_{o}}}\right) \text {. }
$$

The simple notation of the derivative coordinates here is

$$
\begin{aligned}
& \dot{x}_{i_{b}}=\frac{\mathrm{d} x_{i_{b}}}{\mathrm{~d} t}, \\
& \dot{x}_{i_{s_{e}}}=\frac{\mathrm{d} x_{i_{s_{e}}}}{\mathrm{~d} t}, \\
& \dot{x}_{i_{s_{o}}}=\frac{\mathrm{d} x_{i_{s_{o}}}}{\mathrm{~d} t} .
\end{aligned}
$$

Now we define graph manifold. $M$ is a three-manifold. Class $\mathrm{M}$ consists of 3-dimensional, connected, closed, orientable manifold. Associated with every $M$ is its graph $G$. The vertex set $V$ of $G$ is the set of maximal blocks in $M$, and the set $E$ of the oriented edges of $G$ can be identified with the set of boundary components of all maximal blocks. Namely, an edgee $\in E$ is directed from a vertex $x$ to a vertex $y$, if the boundary torus $T_{e} \subset \partial M_{x}$ is attached in $M$ where to the boundary torus $T_{-e} \subset \partial M_{y}$, where the minus sign means reserve edge orientation. The incompressible torus in $M$ that results from gluing the boundary tori $T_{e}$ and $T_{-e}$ will be denoted as $T_{|e|}$. The set of the edges coming out from a vertex is denoted by $\partial_{x}$, and if $e \in \partial_{x}$, then we write $e^{-}=x,(-e)^{+}=x$. 
Definition 3. Let $B$ and $F$ be graphs. A graph $G$ is graph bundle with fiber $F$ over the base graph $B$ if there is a mapping $p: G \longrightarrow B$ which satisfies the following conditions:

(1) It maps adjacent vertices of $G$ to adjacent or identical vertices in $B$

(2) The edges are mapped to edges or collapsed to a vertex

(3) For each vertex $v \in V(B), p^{-1}(v) \cong F$, and for each edge $e \in E(B), p^{-1}(e) \cong K \times F$

A mapping satisfying just the two conditions above is called a graph map. For a given graph $G$, there may be several mappings $p_{i}: G \longrightarrow B_{i}$ with the above properties. In such cases, we write $\left(G, p_{i}, B_{i}\right)$ to avoid confusion. Now we introduce an equivalence relation $\delta^{*}$ defined among the edges of a graph. With this relation can be recognized graph bundles [9].

An induced cycle of four vertices is called a chordless square. With this definition, we can define an auxiliary binary relation $\delta$. For any $e, f \in E(G)$, we set $e \delta f$ if at least one of the following conditions is satisfied:

(1) $e$ and $f$ are opposite edges of a chordless square

(2) $e$ and $f$ are adjacent and there is no chordless square spanned on $e$ and $f$ [9]

By $\delta^{*}$, we denote the reflexive and transitive closure of $\delta$. Since $\delta$ is symmetric, $\delta^{*}$ is an equivalence relation. Any pair of adjacent edges which belong to distinct $\delta^{*}$-equivalence classes spans a chordless square.

If we take graph manifolds instead of $E$ and $M$ manifolds in the bundle definition given in Definition 1 , and a $\pi$ is also a graph map, a graph bundle is obtained when the conditions given in Definition 3 are provided. So, we can obtain the energy equation on a graph bundle. Now we have defined the graphs in superspace. So, we can create the supergraph bundle given by Definition 2. Since graph manifolds are usually taken 3-manifold, we will take the superspace in 3 dimensions in this study. In addition, since the graph manifolds are real, we will take the body parts of the supernumbers as real numbers in this study because if the body part of the number is real, it corresponds to a real number, and if the body part of the number is complex, it corresponds to a complex number.

A vertex in superspace will have the body part and the soul part as well as soul even and soul odd parts. Since graphs are formed by combining vertex and edges, we need to consider soul odd and soul even parts for vertex when defining graphs in superspace. Lines joining points called vertex in graph theory are called edges. In the mechanical system we have introduced to calculate the energy equation during the motion of a moving particle, these vertex points become the points that the object passes through during its motion. Edges also correspond to the paths formed by the movement of the object between these vertex points. It can also be regarded as a vector geometrically because it is a directional line segment. The combination of all the paths that can occur as the direction of motion changes again forms a graph in superspace. $E: X \longrightarrow Y$ ( $X$ and $Y$ are vertex and $E$ is edge), and $(E, X)$ is a graph. We will denote the parity with $(w)$ for the graphs. In superspace, if $|w|=1$, the number is odd, and if $|w|=0$, the number is even. When defining geometric structures in superspace, the sign of odd parts must be negative. This is a necessity for the study to give correct results. Physically, it can be explained with direction. In geometry, the positive direction is counterclockwise direction, and the negative direction is on the contrary of it, that is, clockwise direction. In the graph manifold definition, we said that the minus sign is related to the orientation. Therefore, when defining the graph theory in superspace, the concept of direction should be determined by examining the soul odd and soul even parts of the vertices. A supernumber can consist of body, soul odd and soul even parts or just body, and soul even or soul odd parts. It is related to whether this number is an odd or an even number. Therefore, since the vertex points will be points in the 3 -dimensional superspace we are working with, the movements between soul even and body parts of these vertex points will show the positive direction and the movements between the soul odd parts will show the negative direction. So, the movement between the soul even and body parts of vertex points is picturing counterclockwise direction and the movement between the soul odd parts of vertex points is picturing clockwise direction.

For example, let the vertex point only consist of body and soul even parts. Consider the movement from this point to another vertex point consisting of body and soul odd parts. Since the movement will first be from the body part to the body part, it will occur in the $(+)$ direction (counterclockwise) and then it will happen in the (-) direction (clockwise) because it will be in the direction from the soul even part to the soul odd part. This can be seen in the graph manifold graph given in Figure 1.

$\Gamma\left(V_{*}, E_{*}\right)$ get a graph on the $M$ supermanifold. Here, $V_{*}$ is the set of vertexes and $E_{*}$ is the set of edges. Let any two subsets of $V_{*}$ be $V_{i}, V_{j}$, also

$$
E_{*}=V_{i} \times V_{j}, \quad(i, j=1,2,3) .
$$

According to this, any open subset of $V_{i} \times V_{j}$ is $U_{V_{i} \times V_{j}}$ and an open subset of $R^{m} \times R^{n_{e}} \times R^{n_{o}}$ is $U_{R^{m} \times R^{n_{e}} \times R^{n_{o}}}$. Here $m$ is body dimension, $n_{e}$ is soul even dimension, and $n_{o}$ is soul odd dimension. The coordinate neighborhood system (atlas) can be obtained for the differential structure on a graph manifold in superspace by defining homeomorphisms on these sets as follows:

$$
\beta_{*}: U_{V_{i} \times V_{j}} \longrightarrow U_{R^{m} \times R^{n_{e}} \times R^{n_{o}}}
$$

to be one to one, so the pair $\left(U_{V_{i} \times V_{j}}, \beta_{*}\right)$ is called a supergraph coordinate neighborhood in $\mathrm{M}$. According to this,

(1) $\cup_{V_{i} \times V_{j}} U_{V_{i} \times V_{j}}=M$

(2) For $\forall V_{i_{1}} \times V_{j_{1}}, V_{i_{2}} \times V_{j_{2}}$ to be $V_{i_{1}} \times V_{j_{1}} \cap$ $V_{i_{2}} \times V_{j_{2}} \neq \varnothing$, hence $\beta_{*} o\left(\beta_{*}\right)^{-1}$ function to be differentiable 


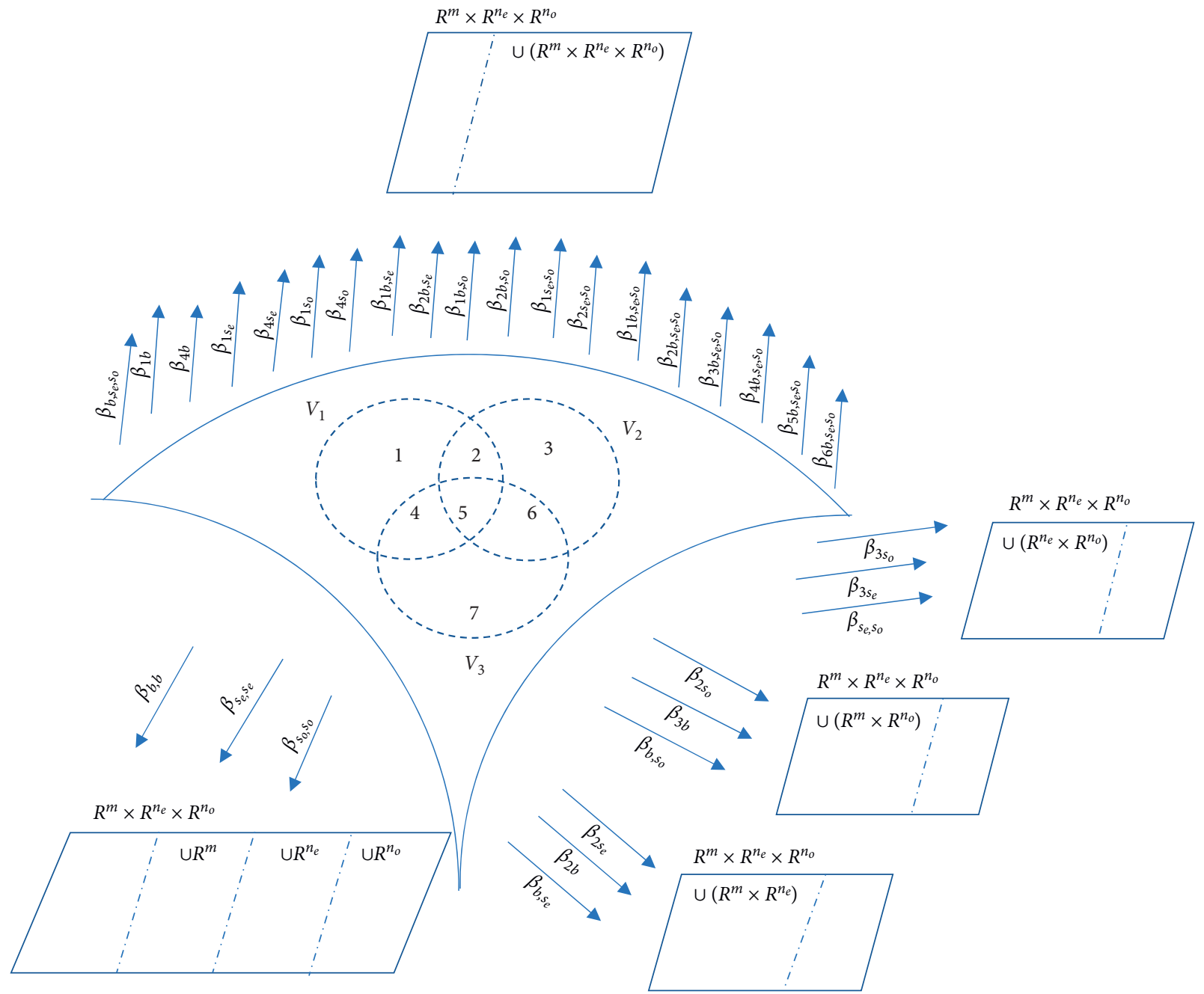

Figure 1: Differentiable structure of a supergraph manifold: definition set and homeomorphisms.

The collection $V_{i} \times V_{j}$ that meets the above conditions is called a supergraph atlas in $M$.

Accordingly, we can picture the differential structure of a supergraph manifold in (Figure 1).

In Figure 1,

$V_{1}$ is the open subset of vertexes that have a body part (b)

$V_{2}$ is the open subset of vertexes that have a soul even part $\left(s_{e}\right)$

$V_{3}$ is the open subset of vertexes that have a soul odd part $\left(s_{o}\right)$

According to this,

(1) In region 1, the $x$ and $y$ vertexes only consist of the body part, i.e., $x=x_{b}$ and $y=y_{b}$

(2) In region 2, the $x$ and $y$ vertexes consist of the body part and soul even part, i.e., $x=x_{b}+x_{s_{e}}$ and $y=y_{b}+y_{s_{e}}$

(3) In region 3, the $x$ and $y$ vertexes only consist of the soul even part, i.e., $x=x_{s_{e}}$ and $y=y_{s_{e}}$
(4) In region 4, the $x$ and $y$ vertexes consist of the body part and soul odd part, i.e., $x=x_{b}+x_{s_{o}}$ and $y=y_{b}+y_{s_{o}}$

(5) In region 5, the $x$ and $y$ vertexes consist of all parts for a supernumber, namely, the body part, soul even part, and soul odd part, i.e., $x=x_{b}+x_{s_{e}}+x_{s_{o}}$ and $y=y_{b}+y_{s_{e}}+y_{s}$

(6) In region 6, the $x$ and $y$ vertexes consist of the soul even part and soul odd part, i.e., $x=x_{s_{e}}+x_{s_{o}}$ and $y=y_{s_{e}}+y_{s_{o}}$

(7) In region 7, the $x$ and $y$ vertexes only consist of the soul odd part, i.e., $x=x_{s_{o}}$ and $y=y_{s_{o}}$

Now we define the homeomorphisms shown in Figure 1 as follows:

$\beta_{b, b}: V_{1}(1) \times V_{1}(1) \longrightarrow \cup R^{m}$ where domain is the edge $e\left(x_{b}, y_{b}\right)$

$\beta_{s_{e}, s_{e}}: V_{2}(3) \times V_{2}(3) \longrightarrow \cup R^{n_{e}}$ where domain is the edge $e\left(x_{s}, y_{s_{e}}\right)$

$\beta_{s_{0}, s_{o}}: V_{3}(7) \times V_{3}(7) \longrightarrow \cup R^{n_{o}}$ where domain is the edge $e\left(x_{s_{o}}, y_{s_{o}}\right)$ 
$\beta_{b, s_{e}}: V_{1} V_{2}(2) \times V_{1} V_{2}(2) \longrightarrow \cup\left(R^{m} \times R^{n_{e}}\right) \quad$ where domain is the edge $e\left(x_{b}+x_{s_{e}}, y_{b}+y_{s_{e}}\right)$

$\beta_{b, s_{o}}: V_{1} V_{3}(4) \times V_{1} V_{3}(4) \longrightarrow \cup\left(R^{m} \times R^{n_{o}}\right) \quad$ where domain is the edge $e\left(x_{b}+x_{s_{o}}, y_{b}+y_{s_{o}}\right)$

$\beta_{s_{e}, s_{o}}: V_{2} V_{3}(6) \times V_{2} V_{3}(6) \longrightarrow \cup\left(R^{n_{e}} \times R^{n_{o}}\right) \quad$ where domain is the edge $e\left(x_{s_{e}}+x_{s_{o}}, y_{s_{e}}+y_{s_{o}}\right)$ $\beta_{b, s_{e}, s_{o}}: V_{1} V_{2} V_{3}(5) \times V_{1} V_{2} V_{3}(6) \stackrel{\cup}{\longrightarrow}\left(R^{m} \times R^{n_{e}} \times\right.$ $\left.R^{n_{o}}\right)$ where domain is the edge $e\left(x_{b}+x_{s_{e}}+x_{s_{o}}\right.$, $\left.y_{b}+y_{s_{e}}+y_{s_{o}}\right)$

Here, thirty-one distinct homeomorphism maps can be defined between the body, soul even, and soul odd parts of the supersets, as follows:

$\beta_{1 b}: V_{1}(1) \times V_{2} V_{3}(6) \longrightarrow \cup\left(R^{m} \times R^{n_{e}} \times R^{n_{o}}\right)$ where domain is the edge $e\left(x_{b}, y_{s_{e}}+y_{s_{o}}\right)$

$\beta_{2 b}: V_{1}(1) \times V_{1} V_{2}(2) \longrightarrow \cup\left(R^{m} \times R^{n_{e}}\right)$ where domain is the edge $e\left(x_{b}, y_{b}+y_{s_{e}}\right)$

$\beta_{3 b}: V_{1}(1) \times V_{1} V_{3}(4) \longrightarrow \cup\left(R^{m} \times R^{n_{o}}\right)$ where domain is the edge $e\left(x_{b}, y_{b}+y_{s_{o}}\right)$

$\beta_{4 b}: V_{1}(1) \times V_{1} V_{2} V_{3}(5) \longrightarrow \cup\left(R^{m} \times R^{n_{e}} \times R^{n_{o}}\right)$

where domain is the edge $e\left(x_{b}, y_{b}+y_{s_{e}}+y_{s_{o}}\right)$

$\beta_{1 s_{e}}: V_{2}(3) \times V_{1} V_{3}(4) \longrightarrow \cup\left(R^{m} \times R^{n_{e}} \times R^{n_{o}}\right)$ where domain is the edge $e\left(x_{s_{e}}, y_{b}+y_{s_{o}}\right)$

$\beta_{2 s_{e}}: V_{2}(3) \times V_{1} V_{2}(2) \stackrel{\longrightarrow}{\longrightarrow}\left(R^{m} \times R^{n_{e}}\right)$ where domain is the edge $e\left(x_{s_{e}}, y_{b}+y_{s_{e}}\right)$

$\beta_{3 s_{e}}: V_{2}(3) \times V_{2} V_{3}(6) \longrightarrow \cup\left(R^{n_{e}} \times R^{n_{o}}\right)$ where domain is the edge $e\left(x_{s_{e}}, y_{s_{e}}+y_{s_{o}}\right)$

$\beta_{4 s_{e}}: V_{2}(3) \times V_{1} V_{2} V_{3}(5) \stackrel{\cup}{\longrightarrow}\left(R^{m} \times R^{n_{e}} \times R^{n_{o}}\right)$

where domain is the edge $e\left(x_{s_{e}}, y_{b}+y_{s_{e}}+y_{s_{o}}\right)$

$\beta_{1 s_{o}}: V_{3}(7) \times V_{1} V_{2}(2) \longrightarrow \cup\left(\stackrel{R^{m}}{\longrightarrow} \times R^{n_{e}} \times R^{n_{o}}\right)$ where domain is the edge $e\left(x_{s_{o}}, y_{b}+y_{s_{e}}\right)$

$\beta_{2 s_{o}}: V_{3}(7) \times V_{1} V_{3}(4) \stackrel{\longrightarrow}{\longrightarrow}\left(R^{m} \times R^{n_{o}}\right)$ where domain is the edge $e\left(x_{s_{o}}, y_{b}+y_{s_{o}}\right)$

$\beta_{3 s_{o}}: V_{3}(7) \times V_{2} V_{3}(6) \longrightarrow \cup\left(R^{n_{e}} \times R^{n_{o}}\right)$ where domain is the edge $e\left(x_{s_{0}}, y_{s_{e}}+y_{s_{0}}\right)$

$\beta_{4 s_{o}}: V_{3}(7) \times V_{1} V_{2} V_{3}(5) \stackrel{\cup}{\longrightarrow}\left(R^{m} \times R^{n_{e}} \times R^{n_{o}}\right)$

where domain is the edge $e\left(x_{s_{o}}, y_{b}+y_{s_{e}}+y_{s_{o}}\right)$

$\beta_{1 b, s_{e}}: V_{1} V_{2}(2) \times V_{3}(7) \longrightarrow \cup\left(R^{m} \times R^{n_{e}} \times R^{n_{o}}\right)$

where domain is the edge $e\left(x_{b}+x_{s_{e}}, y_{s_{o}}\right)$

$\beta_{2 b, s_{e}}: V_{1} V_{2}(2) \times V_{1} V_{2} V_{3}(5) \longrightarrow \cup^{(}\left(R^{m_{o}} \times R^{n_{e}} \times R^{n_{o}}\right)$

where domain is the edge $e\left(x_{b}+x_{s_{e}}, y_{b}+y_{s_{e}}+y_{s_{o}}\right)$

$\beta_{1 b, s_{o}}: V_{1} V_{3}(4) \times V_{2}(3) \longrightarrow \cup\left(R^{m} \times R^{n_{e}} \times R^{n_{o}}\right)$

where domain is the edge $e\left(x_{b}+x_{s_{o}}, y_{s_{e}}\right)$

$\beta_{2 b, s}: V_{1} V_{3}(4) \times V_{1} V_{2} V_{3}(5) \longrightarrow \cup^{\prime}\left(R^{m} \times R^{n_{e}} \times R^{n_{o}}\right)$

where domain is the edge $e\left(x_{b}+x_{s_{o}}, y_{b}+y_{s_{e}}+y_{s_{o}}\right)$

$\beta_{1 s_{e}, s_{o}}: V_{2} V_{3}(6) \times V_{1}(1) \longrightarrow \cup\left(R^{m} \times R^{n_{e}} \times R^{n_{o}}\right)$

where domain is the edge $e\left(x_{s_{e}}+x_{s_{0}}, y_{b}\right)$

$\beta_{2 s_{e}, s_{o}}: V_{2} V_{3}(6) \times V_{1} V_{2} V_{3}(5) \stackrel{\text { 列 }}{\longrightarrow}\left(R^{m} \times R^{n_{e}} \times R^{n_{o}}\right)$

where domain is the edge $e\left(x_{s_{e}}+x_{s_{o}}, y_{b}+y_{s_{e}}+y_{s_{o}}\right)$

$\beta_{1 b, s_{e}, s_{o}}: V_{1} V_{2} V_{3}(5) \times V_{1}(1) \longrightarrow \cup\left(R^{m} \times R^{n_{e}} \times R^{n_{o}^{o}}\right)$

where domain is the edge $e\left(x_{b}+x_{s_{e}}+x_{s_{o}}, y_{b}\right)$

$\beta_{3 b, s_{e}, s_{o}}: V_{1} V_{2} V_{3}(5) \times V_{3}(7) \longrightarrow \cup\left(R^{m} \times R^{n_{e}} \times R^{n_{o}}\right)$

where domain is the edge $e\left(x_{b}+x_{s_{e}}+x_{s_{o}}, y_{s_{o}}\right)$

$\beta_{4 b, s_{e}, s_{o}}: V_{1} V_{2} V_{3}(5) \times V_{1} V_{2}(2) \longrightarrow \cup\left(R^{m} \times R^{n_{e}} \times\right.$

$\left.R^{n_{o}}\right)$ where domain is the edge $e\left(x_{b}+x_{s_{e}}+x_{s_{o}}\right.$, $y_{b}+y_{s_{e}}$ )

$\beta_{5 b, s_{e}, s_{o}}: V_{1} V_{2} V_{3}(5) \times V_{1} V_{3}(4) \longrightarrow \cup\left(R^{m} \times R^{n_{e}} \times\right.$ $\left.R^{n_{o}}\right)$ where domain is the edge $e\left(x_{b}+x_{s_{e}}+x_{s_{o}}\right.$, $\left.y_{b}+y_{s_{o}}\right)$ $\beta_{6 b, s_{e}, s_{o}}: V_{1} V_{2} V_{3}(5) \times V_{2} V_{3}(6) \longrightarrow \cup\left(R^{m} \times R^{n_{e}} \times\right.$ $\left.R^{n_{o}}\right)$ where domain is the edge $e\left(x_{b}+x_{s_{e}}+x_{s_{o}}\right.$, $\left.y_{s_{e}}+y_{s_{o}}\right)$

According to the transformations we have defined above, the edges obtained by combining the vertex on a graph manifold can be depicted as follows. Thus, the graph manifold can be shown in Figure 2. Considering each of the above $\beta$ maps, edges are depicted linearly between vertexes with the same part, and edges between vertexes with different parts are depicted as curves because when combining vertexes with different parts, the coordinate order must be considered (body, soul even, and soul odd). These couplings are shown in Figure 2. The orientations in this graph will be determined as such: in vertexes, in the orientation from even parity to even parity, counterclockwise, that is, positive orientation is taken; in the orientation from even parity to odd parity or from odd parity to even parity, clockwise, that is, negative orientation is taken.

Definition 4. Let $M^{*}$ and $F^{*}$ be supergraph manifolds. A supergraph $G^{*}$ is a supergraph bundle with fiber $F^{*}$ over the base supergraph $M^{*}$ if there is a mapping $\pi^{* g}: G^{*} \longrightarrow M^{*}$ which satisfies the following conditions:

(1) It maps adjacent vertices of $G^{*}$ to adjacent or identical vertices in $M^{*}$ according to the orientation properties for the graph manifold (Figure 2)

(2) Under the same conditions expressed above, the edges are mapped to edges or collapsed to a vertex

(3) For each vertex $x \in V\left(M^{*}\right), \pi^{* g^{-1}}(x) \cong F^{*}$, and for each edge $e \in E\left(M^{*}\right), \pi^{*} g^{-1}(e) \cong K^{*} \times F^{*}$

A mapping satisfying just the two conditions above is called a supergraph map. We can also define this map $\pi^{* g}$ as vertex-edge transformation, and in other words, the values created by the combination of each vertex we receive will turn into the edges formed by the combination of vertexes that these vertexes will correspond with this transformation. The subset $\pi^{* g^{-1}}(e)$ of $G^{*}$ is also called supergraph fiber. $p^{*}$ is also a covering submersion.

The equivalence class defined among the edges can be defined as supergraph bundles. For any $e, f \in E\left(G^{*}\right)$, we set $e \delta^{*} f$ if at least one of the following conditions is satisfied:

(1) $e$ and $f$ are opposite edges between the body, soul even, or soul odd parts of the vertex that have a chordless square

(2) $e$ and $f$ are adjacent according to directions in Figure 2 and there is no chordless square spanned on $e$ and $f$

Since $\delta^{*}$ is symmetric, it is an equivalence relation. Consequently, the triple $\left(G^{*}, \pi^{* g}, M^{*}\right)$ is called a supergraph bundle. The dimensions of supergraph manifolds are as follows:

$$
\begin{aligned}
& \text { boy } M^{*}=m=\left(m_{b}+m_{s_{e}}+m_{s_{o}}\right) \\
& \text { boy } G^{*}=m+n=\left(m_{b}+m_{s_{e}}+m_{s_{o}}+n_{b}+n_{s_{e}}+n_{s_{o}}\right)
\end{aligned}
$$


Get the supergraph coordinate system on the superopen subset

$$
\begin{aligned}
& U_{V_{i} \times V_{j}} \subset G^{*}, \\
& y^{* g}: U_{V_{i} \times V_{j}} \longrightarrow R^{m_{b}+n_{b}} \times R^{m_{s_{e}}+n_{s_{e}}} \times R^{m_{s_{o}}+n_{s_{o}}}, \\
& \operatorname{pr}_{1}^{* g}: R^{m_{b}+n_{b}} \times R^{m_{s_{e}}+n_{s_{e}}} \times R^{m_{s_{o}}+n_{s_{o}}} \longrightarrow R^{m_{b}} \times R^{m_{s_{e}}} \times R^{m_{s_{o}}},
\end{aligned}
$$

$x, y \in U_{V_{i} \times V_{j}}$, where $x$ and $y$ are each edge; for instance, edge $x$ is the combination of vertex $x_{i *} \in V_{i}$ and $x_{j *} \in V_{j}$.

$$
\pi^{* g}(x)=\pi^{* g}(y)=p \Longrightarrow p r_{1}^{* g}\left(y^{* g}(x)\right)=p r_{1}^{* g}\left(y^{* g}(y)\right) \text {. }
$$

If this proposition is true, then $y^{* g}$ is called a supergraph adapted coordinate system.

The representation of this in graph theory is edge lines that can be orientable to the vertex from which they started, namely, point $x$. Since the first jets are identical to tangent vectors, the same identification is made for the first jets. Then, $T G^{*} \cong J \pi^{* g}$. Thence, the necessary jet bundle to install time-dependent mechanical systems for supergraphs on the supergraph bundle $\left(G^{*}, \pi^{* g}, M^{*}\right)$ will be $\left(J \pi^{* g}, \pi^{* g}, M^{*}\right)$. Therefore, the coordinates of a graph jet bundle are expressed as follows, considering the coordinate systems we have given above:

$$
(x, y, u)=\left(x_{b}+x_{s_{e}}+x_{s_{o}}, y_{b}+y_{s_{e}}+y_{s_{o}}, u_{b}+u_{s_{e}}+u_{s_{o}}\right), \quad x \in M^{*}, y \in G^{*}, u \in J \pi^{* g}
$$

The coordinate $u$ corresponds to an oriented edge $e$ that can be written as $1(\mathrm{e})=\mathrm{x}$ for the initial vertex. For $x, y$ vertexes, $u=(x, y) \Longrightarrow y-x=u$, that is, $u$ is also a directional line segment (geometrically a vector) from $x$ to $y$ which is corresponding to an edge. In graph theory, this coordinate corresponds to the derivative coordinate for the jet bundle. According to the derivative coordinate definition, $u$ component can be expressed as follows, but for the graph manifold structure, the explanations given next to it will be taken into consideration:

$$
\begin{aligned}
& \frac{\partial y_{b}}{\partial x_{b}}=u_{b}: \text { it corresponds to the } e\left(x_{b}, y_{b}\right) \text { edges which are the combination of } x_{b} \text { and } y_{b} \text { vertexes, } \\
& \frac{\partial y_{s_{e}}}{\partial x_{s_{e}}}=u_{s_{e}} \text { it corresponds to the } e\left(x_{s_{e}}, y_{s_{e}}\right) \text { edges which are the combination of } x_{s_{e}} \text { and } y_{s_{e}} \text { vertexes, } \\
& \frac{\partial y_{s_{o}}}{\partial x_{s_{o}}}=u_{s_{o}}: \text { it corresponds to the } e\left(x_{s_{o}}, y_{s_{o}}\right) \text { edges which are the combination of } x_{s_{o}} \text { and } y_{s_{o}} \text { vertexes. }
\end{aligned}
$$

Here, as we explained in the bundle definition, the derivative of the body part with respect to the body part, the derivative of the soul even part with respect to the soul even part, and the derivative of the soul odd part with respect to the soul odd part will be taken. Mixed derivatives cannot be taken between parts. The components of edge coordinates (derivative coordinates) should also be expressed in this way. For example, when we get the following map, $\beta_{3 b, s_{e}, s_{o}}: V_{1} V_{2} V_{3}(5) \times V_{3}(7) \longrightarrow \cup\left(R^{m} \times R^{n_{e}} \times R^{n_{o}}\right)$ where domain is the edge $e\left(x_{b}+x_{s_{e}}+x_{s_{o}}, y_{s_{o}}\right)$, a coordinate of the graph jet bundle will take the form

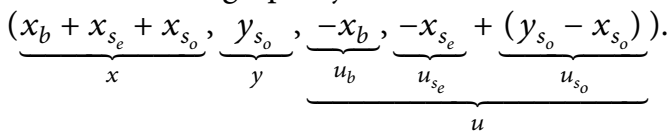

Definition 5. $\Gamma(V, E)$ be a graph with finite vertex set $V$ and edge set $E$. Given an oriented edge $e$, that can be written as $1(\mathrm{e})=\mathrm{x}$ for the initial vertex. $\operatorname{Star}(x)=\{e \mid \operatorname{1t}(\mathrm{e}) \mathrm{n}=\mathrm{qx}\}$ is the tangent space of the graph manifold at a point $x$. Elements of this space are called tangent vectors [6].

Geometrically, a tangent vector is a vector that is tangent to a curve or surface at a given point. Tangent vectors are described in the differential geometry of curves in the context of curves in $R^{n}$. More generally, tangent vectors are elements of a tangent space of a differentiable manifold. Consider a fixed point $X$ and a moving point $P$ on a curve. As point $P$ moves toward $X$, the vector from $X$ to $P$ approaches the tangent vector at $X$ (Figure 3 ). The line that contains the tangent vector is the tangent line. In this work, we will denote the tangent space of the graph manifold, that is, the set Star $(x)$ with TS. Additionally, a tangent vector $v_{p}$ on the manifold $M$ is a map $v_{p}: C^{\infty}(M) \longrightarrow R(p \in M)$. We have special tangent vectors $\left\{\left(\partial / \partial x^{k}\right)(p), 1 \leq k \leq 3\right\}$ (called the partial derivatives $),\left.\quad\left(\partial / \partial x^{k}\right)\right|_{p}: C^{\infty}(M) \longrightarrow R,\left.\quad\left(\partial / \partial x^{k}\right)\right|_{p}(f)=$ $\left.\left(\partial\left(f \circ \varnothing^{-1}\right) / \partial u^{k}\right)\right|_{\varnothing(p)}((U, \varnothing)$ local coordinate system for 
$M$ at $p) .\left\{\left(\partial / \partial x^{k}\right)(p)\right\}$ tangent vectors system is the base for the tangent manifold TM.

The base for the graph tangent manifold TM is denoted in the same way, and this vector system is denoted as $\left\{\left(\partial / \partial x_{b}\right),\left(\partial / \partial x_{s_{e}}\right),\left.\left(\partial / \partial x_{s_{o}}\right)\right|_{p}\right\}$.

A vector bundle is a special class of fiber bundle in which the fiber is a vector space $V$. If $(E, f, B)$ is a bundle with fiber $R^{n}$, to be a vector bundle, all of the fibers $f^{-1}(x)(x \in B)$ need to have a coherent vector space structure. Also, a vector bundle is a total space $E$ along with a surjective map $f: E \longrightarrow B$ to a base manifold $B$. Any fiber $f^{-1}(x)$ is a vector space isomorphic to $V$. So, each point $x$ of the manifold $M$ corresponds to a vector. In graph theory, it is an orientable graph for each $x_{i}$ vertex, formed by vectors accepted as edges between these vertices. For example, in Figure 2, the system of edges formed between for a finite number of some paths $x$ and $y$ vertices on the graph manifold becomes a vector bundle.

While defining the geometric structures in Section 3 in graph theory, we will make use of the definitions and explanations given in this section.

\section{Lagrangian Mechanical Systems for Superspace with Superjet Bundle and Supergraph Bundle}

In this section, we will first obtain Lagrangian mechanical systems with superjet bundle structure. Thus, we will have time-dependent Lagrange energy equations in superspace.
Then we will construct these geometric structures for supergraph bundle. We will compare the time-dependent Lagrangian energy equation obtained from the solution of the mechanical system for graph bundle coordinates with the previous equation. Thus, the Lagrangian energy equation will be obtained for the graph bundle.

Definition 6. Let $J$ be a tensor field of type first-order covariant and first-order contravariant such that $J: T\left(J^{1} E^{*}\right) \longrightarrow T\left(J^{1} E^{*}\right)$ by

$$
\begin{aligned}
J\left(\frac{\partial}{\partial x_{i_{b}}}\right) & =\frac{\partial}{\partial \dot{x}_{i_{b}}} \\
J\left(\frac{\partial}{\partial x_{i_{s_{e}}}}\right) & =\frac{\partial}{\partial \dot{x}_{i_{s_{e}}}} \\
J\left(\frac{\partial}{\partial x_{i_{s_{o}}}}\right) & =\frac{\partial}{\partial \dot{x}_{i_{s_{o}}}} \\
J\left(\frac{\partial}{\partial \dot{x}_{i_{b}}}\right) & =J\left(\frac{\partial}{\partial \dot{x}_{i_{s_{e}}}}\right) J\left(\frac{\partial}{\partial \dot{x}_{i_{s_{o}}}}\right)=0 \\
J\left(\frac{\partial}{\partial t}\right) & =\dot{x}_{i_{b}} \frac{\partial}{\partial \dot{x}_{i_{b}}}+\dot{x}_{i_{s_{e}}} \frac{\partial}{\partial \dot{x}_{i_{s_{e}}}} \dot{x}_{i_{s_{o}}} \frac{\partial}{\partial \dot{x}_{i_{s_{o}}}}
\end{aligned}
$$

We will write this tensor field in coordinates as follows:

$$
J=\left(\mathrm{d} x_{i_{b}}+\dot{x}_{i_{b}} \mathrm{~d} t_{b}\right) \times \frac{\partial}{\partial \dot{x}_{i_{b}}}+\left(\mathrm{d} x_{i_{s_{e}}}+\dot{x}_{i_{s_{e}}} \mathrm{~d} t_{s_{e}}\right) \times \frac{\partial}{\partial \dot{x}_{i_{s_{e}}}}-\left(\mathrm{d} x_{i_{s_{o}}}+\dot{x}_{i_{s_{o}}} \mathrm{~d} t_{s_{o}}\right) \times \frac{\partial}{\partial \dot{x}_{i_{s_{o}}}}
$$

This tensor field is named as super almost tangent structure and provides the condition

$$
J^{2}=0 \text {. }
$$

Definition 7. A semispray in superspace is a vector field over the total manifold $E^{*}$ and defined as follows:

$$
\varepsilon=\frac{\partial}{\partial t_{b}}+\frac{\partial}{\partial t_{s_{e}}}-\frac{\partial}{\partial t_{s_{o}}}+\dot{x}_{i_{b}} \frac{\partial}{\partial x_{i_{b}}}+\dot{x}_{i_{s_{e}}} \frac{\partial}{\partial x_{i_{s_{e}}}}-\dot{x}_{i_{s_{o}}} \frac{\partial}{\partial x_{i_{s_{o}}}}+\varepsilon_{i_{b}} \frac{\partial}{\partial \dot{x}_{i_{b}}}+\varepsilon_{s_{e}} \frac{\partial}{\partial \dot{x}_{i_{s_{e}}}}-\varepsilon_{s_{o}} \frac{\partial}{\partial \dot{x}_{i_{s_{o}}}}
$$

If we calculate the $J$-directional derivative $J(\varepsilon)$ of the semispray $\varepsilon$, we can obtain the Liouville vector field $V$ :

$$
V=J(\varepsilon)=2 \dot{x}_{i_{b}} \frac{\partial}{\partial x_{i_{b}}}+2 \dot{x}_{i_{s_{e}}} \frac{\partial}{\partial x_{i_{s_{e}}}}-2 \dot{x}_{i_{s_{o}}} \frac{\partial}{\partial x_{i_{s_{o}}}}
$$

To set up and solve the Lagrangian mechanical system, we have to create Poincaré-Cartan 1-form and Poincaré-Cartan 2-form in superspace. Firstly, we must write the differential operator $d$ with supercoordinate system as follows:

$$
d=\frac{\partial}{\partial t_{b}} \mathrm{~d} t_{b}+\frac{\partial}{\partial t_{s_{e}}} \mathrm{~d} t_{s_{e}}-\frac{\partial}{\partial t_{s_{o}}} \mathrm{~d} t_{s_{o}}+\frac{\partial}{\partial x_{i_{b}}} \mathrm{~d} \dot{x}_{i_{b}}+\frac{\partial}{\partial x_{i_{s_{e}}}} \mathrm{~d} \dot{x}_{i_{s_{e}}}-\frac{\partial}{\partial x_{i_{s_{o}}}} \mathrm{~d} \dot{x}_{i_{s_{o}}}+\frac{\partial}{\partial \dot{x}_{i_{b}}} \mathrm{~d} \dot{x}_{i_{b}}+\frac{\partial}{\partial \dot{x}_{i_{s_{e}}}} \mathrm{~d} \dot{x}_{i_{s_{e}}}-\frac{\partial}{\partial \dot{x}_{i_{s_{o}}}} \mathrm{~d} \dot{x}_{i_{s_{o}}}
$$




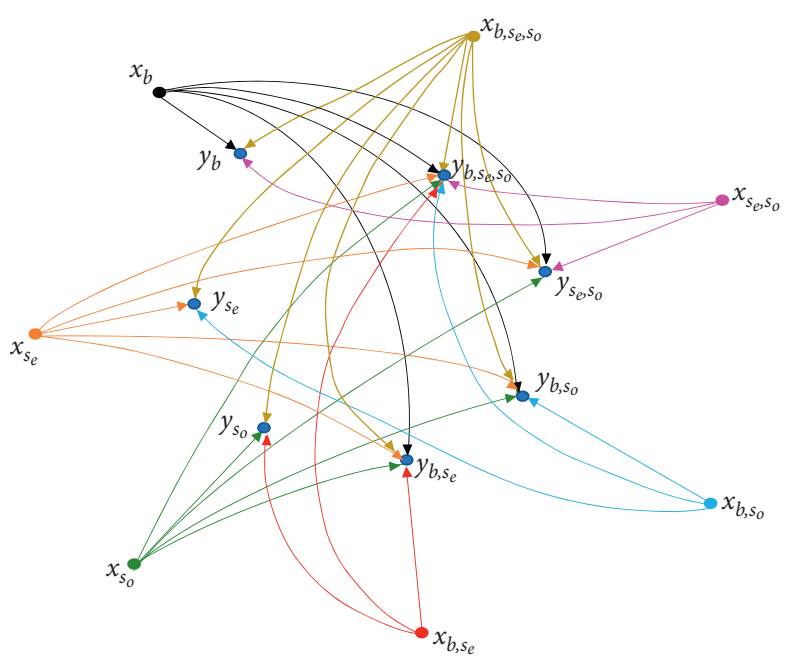

Figure 2: Figure of a graph manifold: vertexes and edges.

Now let us form the Poincaré-Cartan 1-form $\alpha_{L}$ first,

$$
\alpha_{L}=d_{J} L+L \mathrm{~d} t=\dot{x}_{i_{b}} \frac{\partial L_{b}}{\partial \dot{x}_{i_{b}}} \mathrm{~d} t_{b}+\dot{x}_{i_{e}} \frac{\partial L_{s_{e}}}{\partial \dot{x}_{i_{s_{e}}}} \mathrm{~d} t_{s_{e}}-\dot{x}_{i_{s_{o}}} \frac{\partial L_{s_{o}}}{\partial \dot{x}_{i_{s_{o}}}} \mathrm{~d} t_{s_{o}}+\frac{\partial L_{b}}{\partial \dot{x}_{i_{b}}} \mathrm{~d} \dot{x}_{i_{b}}+\frac{\partial L_{s_{e}}}{\partial \dot{x}_{i_{s_{e}}}} \mathrm{~d} \dot{x}_{i_{s_{e}}}-\frac{\partial L_{s_{o}}}{\partial \dot{x}_{i_{s_{o}}}} \mathrm{~d} \dot{x}_{i_{s_{o}}}+L_{b} \mathrm{~d} t_{b}+L_{s_{e}} \mathrm{~d} t_{s_{e}}-L_{s_{o}} \mathrm{~d} t_{s_{o}}
$$

By differentiating Poincaré-Cartan 1-form, we get Poincaré-Cartan 2-form as follows:

$$
\begin{aligned}
& \Omega_{L}=d d_{J} L+\mathrm{d} L \wedge \mathrm{d} t=\left(\mathrm{d} x_{i_{b}} \wedge \mathrm{d} t_{b}\right)\left(-\frac{\partial^{2} L_{b}}{\partial t_{b} \partial \dot{x}_{i_{b}}}+\dot{x}_{i_{b}} \frac{\partial^{2} L_{b}}{\partial x_{i_{b}} \partial \dot{x}_{i_{b}}}+\frac{\partial L_{b}}{\partial x_{i_{b}}}\right) \\
& +\left(\mathrm{d} x_{i_{s_{e}}} \wedge \mathrm{d} t_{s_{e}}\right)\left(-\frac{\partial^{2} L_{s_{e}}}{\partial t_{s_{e}} \partial \dot{x}_{i_{s_{e}}}}+\dot{x}_{i_{s_{e}}} \frac{\partial^{2} L_{s_{e}}}{\partial x_{i_{s_{e}}} \partial \dot{x}_{i_{s_{e}}}}+\frac{\partial L_{s_{e}}}{\partial x_{i_{s_{e}}}}\right)+\left(\dot{\mathrm{d} x} x_{i_{s_{o}}} \wedge \mathrm{d} t_{s_{o}}\right)\left(-\frac{\partial^{2} L_{s_{o}}}{\partial t_{s_{o}} \partial \dot{x}_{i_{s_{o}}}}+\dot{x}_{i_{s_{e}}} \frac{\partial^{2} L_{s_{o}}}{\partial x_{i_{s_{o}}} \partial \dot{x}_{i_{s_{o}}}}+\frac{\partial L_{s_{o}}}{\partial x_{i_{s_{o}}}}\right) \\
& +\left(\mathrm{d} \dot{x}_{i_{b}} \wedge \mathrm{d} t_{b}\right)\left(\dot{x}_{i_{b}} \frac{\partial^{2} L_{b}}{\partial \dot{x}_{i_{b}} \partial \dot{x}_{i_{b}}}+\frac{\partial L_{b}}{\partial \dot{x}_{i_{b}}}\right)+\left(\mathrm{d} \dot{x}_{i_{s_{e}}} \wedge \mathrm{d} t_{s_{e}}\right)\left(\dot{x}_{i_{s_{e}}} \frac{\partial^{2} L_{s_{e}}}{\partial \dot{x}_{i_{s_{e}}} \partial \dot{x}_{i_{s_{e}}}}+\frac{\partial L_{s_{e}}}{\partial \dot{x}_{i_{s_{e}}}}\right)+\left(\mathrm{d} \dot{x}_{i_{s_{o}}} \wedge \mathrm{d} t_{s_{o}}\right)\left(\dot{x}_{i_{s_{o}}} \frac{\partial^{2} L_{s_{o}}}{\partial \dot{x}_{i_{s_{o}}}} \partial \dot{x}_{i_{s_{o}}}+\frac{\partial L_{s_{o}}}{\partial \dot{x}_{i_{s_{o}}}}\right) \\
& +\left(\mathrm{d} \dot{x}_{i_{b}} \wedge \mathrm{d} x_{i_{b}}\right)\left(\frac{\partial^{2} L_{b}}{\partial \dot{x}_{i_{b}} \partial \dot{x}_{i_{b}}}\right)+\left(\mathrm{d} \dot{x}_{i_{s_{e}}} \wedge \mathrm{d} x_{i_{s_{e}}}\right)\left(\frac{\partial^{2} L_{s_{e}}}{\partial \dot{x}_{i_{s_{e}}} \partial \dot{x}_{i_{s_{e}}}}\right)+\left(\mathrm{d} \dot{x}_{i_{s_{o}}} \wedge \mathrm{d} x_{i_{s_{o}}}\right)\left(\frac{\partial^{2} L_{s_{o}}}{\partial \dot{x}_{i_{s_{o}}} \partial \dot{x}_{i_{s_{o}}}}\right) \text {. }
\end{aligned}
$$

Theorem 1. The Euler-Lagrange energy equation in superspace will be obtained as follows:

$$
\dot{x}_{i_{b}} \frac{\partial^{2} L_{b}}{\partial t_{b} \partial \dot{x}_{i_{b}}}+\dot{x}_{i_{s_{e}}} \frac{\partial^{2} L_{s_{e}}}{\partial t_{s_{e}} \partial \dot{x}_{i_{s_{e}}}}+\dot{x}_{i_{s_{o}}} \frac{\partial^{2} L_{s_{o}}}{\partial t_{s_{o}} \partial \dot{x}_{i_{s_{o}}}}-\dot{x}_{i_{b}} \frac{\partial L_{b}}{\partial x_{i_{b}}}-\dot{x}_{i_{s_{e}}} \frac{\partial L_{s_{e}}}{\partial x_{i_{s_{e}}}}-\dot{x}_{i_{s_{o}}} \frac{\partial L_{s_{o}}}{\partial x_{i_{s_{o}}}}=0
$$


Proof. Euler-Lagrange energy is obtained by solving the dynamical equation $i_{\varepsilon} \Omega_{L}=\Omega_{L}(\varepsilon)=0$. Thus, let us set up and solve this dynamical equation using the geometric concepts we have defined above according to the supercoordinate system in superspace:

$$
\begin{aligned}
& \Omega_{L}(\varepsilon)=\left(-\frac{\partial^{2} L_{b}}{\partial t_{b} \partial \dot{x}_{i_{b}}}+\dot{x}_{i_{b}} \frac{\partial^{2} L_{b}}{\partial x_{i_{b}} \partial \dot{x}_{i_{b}}}+\frac{\partial L_{b}}{\partial x_{i_{b}}}+\dot{\varepsilon}_{i_{b}} \frac{\partial^{2} L_{b}}{\partial \dot{x}_{i_{b}} \partial \dot{x}_{i_{b}}}\right) \mathrm{d} x_{i_{b}}+\left(-\frac{\partial^{2} L_{s_{e}}}{\partial t_{s_{e}} \partial \dot{x}_{i_{s_{e}}}}+\dot{x}_{i_{s_{e}}} \frac{\partial^{2} L_{s_{e}}}{\partial x_{i_{s_{e}}} \partial \dot{x}_{i_{e}}}+\frac{\partial L_{s_{e}}}{\partial x_{i_{s_{e}}}}+\dot{\varepsilon}_{i_{s_{e}}} \frac{\partial^{2} L_{s_{e}}}{\partial \dot{x}_{i_{s_{e}}} \partial \dot{x}_{i_{s_{e}}}}\right) \mathrm{d} x_{i_{s_{e}}} \\
& +\left(\frac{\partial^{2} L_{s_{o}}}{\partial t_{s_{o}} \partial \dot{x}_{i_{s_{o}}}}-\dot{x}_{i_{s_{e}}} \frac{\partial^{2} L_{s_{o}}}{\partial x_{i_{s_{o}}} \partial \dot{x}_{i_{s_{o}}}}-\frac{\partial L_{s_{o}}}{\partial x_{i_{s_{o}}}}-\dot{\varepsilon}_{i_{s_{o}}} \frac{\partial^{2} L_{s_{o}}}{\partial \dot{x}_{i_{s_{o}}} \partial \dot{x}_{i_{s_{o}}}}\right) \mathrm{d} x_{i_{s_{o}}} \\
& +\left(\dot{x}_{i_{b}} \frac{\partial^{2} L_{b}}{\partial t_{b} \partial \dot{x}_{i_{b}}}-\left(\dot{x}_{i_{b}}\right)^{2} \frac{\partial^{2} L_{b}}{\partial x_{i_{b}} \partial \dot{x}_{i_{b}}}-\dot{x}_{i_{b}} \frac{\partial L_{b}}{\partial x_{i_{b}}}-\dot{x}_{i_{b}} \dot{\varepsilon}_{i_{b}} \frac{\partial^{2} L_{b}}{\partial \dot{x}_{i_{b}} \partial \dot{x}_{i_{b}}}-\dot{\varepsilon}_{i_{b}} \frac{\partial L_{b}}{\partial \dot{x}_{i_{b}}}\right) \mathrm{d} t_{b} \\
& +\left(\dot{x}_{i_{s_{e}}} \frac{\partial^{2} L_{s_{e}}}{\partial t_{s_{e}} \partial \dot{x}_{i_{s_{e}}}}-\left(\dot{x}_{i_{s_{e}}}\right)^{2} \frac{\partial^{2} L_{s_{e}}}{\partial x_{i_{s_{e}}} \partial \dot{x}_{i_{s_{e}}}}-\dot{x}_{i_{s_{e}}} \frac{\partial L_{s_{e}}}{\partial x_{i_{s_{e}}}}-\dot{x}_{i_{s_{e}}} \dot{\varepsilon}_{s_{s_{e}}} \frac{\partial^{2} L_{s_{e}}}{\partial \dot{x}_{s_{s_{e}}} \partial \dot{x}_{i_{s_{e}}}}-\dot{\varepsilon}_{i_{s_{e}}} \frac{\partial L_{s_{e}}}{\partial \dot{x}_{i_{s_{e}}}}\right) \mathrm{d} t_{s_{e}} \\
& +\left(-\dot{x}_{i_{s_{o}}} \frac{\partial^{2} L_{s_{o}}}{\partial t_{s_{o}} \partial \dot{x}_{i_{s_{o}}}}+\left(\dot{x}_{i_{s_{o}}}\right)^{2} \frac{\partial^{2} L_{s_{o}}}{\partial x_{i_{s_{o}}} \partial \dot{x}_{i_{s_{o}}}}+\dot{x}_{i_{s_{o}}} \frac{\partial L_{s_{o}}}{\partial x_{i_{s_{o}}}}+\dot{x}_{i_{s_{o}}} \dot{\varepsilon}_{i_{s_{o}}} \frac{\partial^{2} L_{s_{o}}}{\partial \dot{x}_{i_{s_{o}}} \partial \dot{x}_{i_{s_{o}}}}+\dot{\varepsilon}_{i_{s_{o}}} \frac{\partial L_{s_{o}}}{\partial \dot{x}_{s_{s_{o}}}}\right) \mathrm{d} t_{s_{o}} \\
& +\left(\dot{x}_{i_{b}} \frac{\partial^{2} L_{b}}{\partial \dot{x}_{i_{b}} \partial \dot{x}_{i_{b}}}+\frac{\partial L_{b}}{\partial \dot{x}_{i_{b}}}-\dot{x}_{i_{b}} \frac{\partial^{2} L_{b}}{\partial \dot{x}_{i_{b}} \partial \dot{x}_{i_{b}}}\right) \mathrm{d} \dot{x}_{i_{b}}+\left(\dot{x}_{i_{s_{e}}} \frac{\partial^{2} L_{s_{e}}}{\partial \dot{x}_{i_{e}} \partial \dot{x}_{i_{s_{e}}}}+\frac{\partial L_{s_{e}}}{\partial \dot{x}_{i_{s_{e}}}}-\dot{x}_{i_{s_{e}}} \frac{\partial^{2} L_{s_{e}}}{\partial \dot{x}_{i_{s_{e}}} \partial \dot{x}_{i_{s_{e}}}}\right) \mathrm{d} \dot{x}_{i_{s_{e}}} \\
& +\left(-\dot{x}_{i_{s_{o}}} \frac{\partial^{2} L_{s_{o}}}{\partial \dot{x}_{i_{s_{o}}} \partial \dot{x}_{i_{s_{o}}}}-\frac{\partial L_{s_{o}}}{\partial \dot{x}_{i_{s_{o}}}}+\dot{x}_{i_{s_{o}}} \frac{\partial^{2} L_{s_{s_{o}}}}{\partial \dot{x}_{s_{s_{o}}} \partial \dot{x}_{s_{s_{o}}}}\right) \mathrm{d} \dot{x}_{i_{s_{o}}}
\end{aligned}
$$

By equalizing equation (29) to zero, then the following equations are obtained:

$$
\begin{aligned}
& \text { (1) }-\frac{\partial^{2} L_{b}}{\partial t_{b} \partial \dot{x}_{i_{b}}}+\frac{\partial}{\partial x_{i_{b}}}\left(\dot{x}_{i_{b}} \frac{\partial L_{b}}{\partial \dot{x}_{i_{b}}}+L_{b}\right)+\frac{\partial}{\partial \dot{x}_{i_{b}}}\left(\dot{\varepsilon}_{i_{b}} \frac{\partial L_{b}}{\partial \dot{x}_{i_{b}}}\right)=0, \\
& \text { (2) }-\frac{\partial^{2} L_{s_{e}}}{\partial t_{s_{e}} \partial \dot{x}_{i_{s_{e}}}}+\frac{\partial}{\partial x_{i_{s_{e}}}}\left(\dot{x}_{i_{s_{e}}} \frac{\partial L_{s_{e}}}{\partial \dot{x}_{i_{s_{e}}}}+L_{s_{e}}\right)+\frac{\partial}{\partial \dot{x}_{i_{s_{e}}}}\left(\dot{\varepsilon}_{i_{s_{e}}} \frac{\partial L_{s_{e}}}{\partial \dot{x}_{i_{s_{e}}}}\right)=0 \text {, } \\
& \text { (3) } \frac{\partial^{2} L_{s_{o}}}{\partial t_{s_{o}} \partial \dot{x}_{i_{s_{o}}}}-\frac{\partial}{\partial x_{i_{s_{o}}}}\left(\dot{x}_{i_{s_{o}}} \frac{\partial L_{s_{o}}}{\partial \dot{x}_{s_{s_{o}}}}+L_{s_{o}}\right)-\frac{\partial}{\partial \dot{x}_{i_{s_{o}}}}\left(\dot{\varepsilon}_{i_{s_{o}}} \frac{\partial L_{s_{o}}}{\partial \dot{x}_{i_{s_{o}}}}\right)=0 \text {, } \\
& \text { (4) } \dot{x}_{i_{b}} \frac{\partial^{2} L_{b}}{\partial t_{b} \partial \dot{x}_{i_{b}}}-\dot{x}_{i_{b}} \frac{\partial}{\partial x_{i_{b}}}\left(\dot{x}_{i_{b}} \frac{\partial L_{b}}{\partial \dot{x}_{i_{b}}}+L_{b}\right)-\dot{\varepsilon}_{i_{b}} \frac{\partial}{\partial \dot{x}_{i_{b}}}\left(\dot{x}_{i_{b}} \frac{\partial L_{b}}{\partial \dot{x}_{i_{b}}}+L_{b}\right)=0 \text {, } \\
& \text { (5) } \dot{x}_{i_{s_{e}}} \frac{\partial^{2} L_{s_{e}}}{\partial t_{s_{e}} \partial \dot{x}_{i_{s_{e}}}}-\dot{x}_{i_{s_{e}}} \frac{\partial}{\partial x_{i_{s_{e}}}}\left(\dot{x}_{i_{s_{e}}} \frac{\partial L_{s_{e}}}{\partial \dot{x}_{i_{s_{e}}}}+L_{s_{e}}\right)-\dot{\varepsilon}_{i_{s_{e}}} \frac{\partial}{\partial \dot{x}_{i_{s_{e}}}}\left(\dot{x}_{i_{s_{e}}} \frac{\partial L_{s_{e}}}{\partial \dot{x}_{i_{s_{e}}}}+L_{s_{e}}\right)=0 \text {, } \\
& \text { (6) }-\dot{x}_{i_{s_{o}}} \frac{\partial^{2} L_{s_{o}}}{\partial t_{s_{o}} \partial \dot{x}_{i_{s_{o}}}}+\dot{x}_{i_{s_{o}}} \frac{\partial}{\partial x_{i_{s_{o}}}}\left(\dot{x}_{i_{s_{o}}} \frac{\partial L_{s_{o}}}{\partial \dot{x}_{i_{s_{o}}}}+L_{s_{o}}\right)+\dot{\varepsilon}_{i_{s_{o}}} \frac{\partial}{\partial \dot{x}_{i_{s_{o}}}}\left(\dot{x}_{i_{s_{o}}} \frac{\partial L_{s_{o}}}{\partial \dot{x}_{i_{s_{o}}}}+L_{s_{o}}\right)=0 \text {, } \\
& \text { (7) }\left(\dot{x}_{i_{b}} \frac{\partial^{2} L_{b}}{\partial \dot{x}_{i_{b}} \partial \dot{x}_{i_{b}}}+\frac{\partial L_{b}}{\partial \dot{x}_{i_{b}}}-\dot{x}_{i_{b}} \frac{\partial^{2} L_{b}}{\partial \dot{x}_{i_{b}} \partial \dot{x}_{i_{b}}}\right)=0, \\
& \text { (8) }\left(\dot{x}_{i_{s_{e}}} \frac{\partial^{2} L_{s_{e}}}{\partial \dot{x}_{s_{e}} \partial \dot{x}_{i_{s_{e}}}}+\frac{\partial L_{s_{e}}}{\partial \dot{x}_{i_{s_{e}}}}-\dot{x}_{i_{s_{e}}} \frac{\partial^{2} L_{s_{s_{e}}}}{\partial \dot{x}_{i_{e}} \partial \dot{x}_{i_{s_{e}}}}\right)=0 \text {, } \\
& \text { (9) }\left(-\dot{x}_{i_{s_{o}}} \frac{\partial^{2} L_{s_{o}}}{\partial \dot{x}_{i_{s_{o}}} \partial \dot{x}_{i_{s_{o}}}}-\frac{\partial L_{s_{o}}}{\partial \dot{x}_{i_{s_{o}}}}+\dot{x}_{i_{s_{o}}} \frac{\partial^{2} L_{s_{o}}}{\partial \dot{x}_{i_{s_{o}}} \partial \dot{x}_{i_{s_{o}}}}\right)=0 \text {. }
\end{aligned}
$$




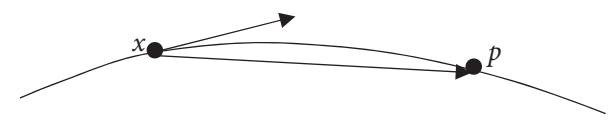

Figure 3: Tangent vector: the vector from $X$ to $P$.

Equation (30) is a system of nonlinear equations. For the solution of this nonlinear equation system, some special conditions are required. Among the different assumptions made, the most appropriate conditions for the general solution of the Lagrangian energy equation are determined as follows:

$$
\begin{aligned}
& \dot{\varepsilon}_{i_{b}}=-\dot{x}_{i_{b}}, \\
& \dot{\varepsilon}_{i_{s_{e}}}=-\dot{x}_{i_{s_{e}}}, \\
& \dot{\varepsilon}_{i_{s_{o}}}=-\dot{x}_{i_{s_{o}}} .
\end{aligned}
$$

We have seen that the general structure of the Lagrange equation shows similarities in different spaces in our previous articles. For this reason, the minus sign here has been accepted in order to create the similarity with the general energy equation, and another reason for this comes from the concept of parity in superspace. As a result of our study, it can be said that the choice of derivative coordinates on the coordinate system of the base manifold will be the most appropriate choice for the solution. These conditions are written in equation (30). Then, a common solution of all these equations is sought. This solution cannot be determined at random. For all equations, a linear relation is determined that will give the most appropriate solution to the standard structure of the energy equation. Among the many linear relations that can be formed for solution, the most accurate relation as shown in the following equation is taken:

$$
-\dot{x}_{i_{b}}(1)-\dot{x}_{i_{s_{e}}}(2)+\dot{x}_{i_{s_{o}}}(3)+(4)+(5)-(6)-\dot{x}_{i_{b}}(7)-\dot{x}_{i_{s_{e}}}(8)+\dot{x}_{i_{s_{o}}}(9)=0 \text {. }
$$

Unlike the conditions we present for the solution here, the signs between the body, even, and odd coordinates are opposite. The necessity of working by taking a sign change originating from parity in the odd part in the superspace is seen in our study. When the above solution is made, the following equation is obtained:

$$
\dot{x}_{i_{b}} \frac{\partial^{2} L_{b}}{\partial t_{b} \partial \dot{x}_{i_{b}}}+\dot{x}_{i_{s_{e}}} \frac{\partial^{2} L_{s_{e}}}{\partial t_{s_{e}} \partial \dot{x}_{i_{s_{e}}}}+\dot{x}_{i_{s_{o}}} \frac{\partial^{2} L_{s_{o}}}{\partial t_{s_{o}} \partial \dot{x}_{i_{s_{o}}}}-\dot{x}_{i_{b}} \frac{\partial L_{b}}{\partial x_{i_{b}}}-\dot{x}_{i_{s_{e}}} \frac{\partial L_{s_{e}}}{\partial x_{i_{s_{e}}}}-\dot{x}_{i_{s_{o}}} \frac{\partial L_{s_{o}}}{\partial x_{i_{s_{o}}}}=0 .
$$

This equation is called the Euler-Lagrange energy equation in superspace.

Now, we will form the mechanical system with supergraph bundle coordinates. When doing this, we will use the bundle structure, coordinate system, and properties for supergraph bundle that we obtained in Section 2. Here, just differently, we have to work with time because we are studying to obtain the time-dependent Lagrangian energy equation. Actually, the coordinate $u$ corresponds to an edge between $x$ and $y$ vertexes, $u=(x, y)$. On this edge, which we can think of geometrically as a vector from $x$ to $y$, the moving particle performs this motion in a time interval $t$. So physically time will be a natural parameter in the 3-dimensional supergraph bundle that we are working with. The coordinates of the supergraph bundle are the same as (19), and only the time relation will be considered. Since only the explication of the edge coordinate is different in bundle coordinates, it will be sufficient to get the geometric structures that form the mechanical system that we have obtained in the first part of Section 3 according to the graph bundle coordinates for the graph bundle because the proofs are similar.

The super almost tangent structure for supergraph bundle is as follows:

$$
J=\left(\mathrm{d} y_{b}+u_{b} \mathrm{~d} x_{b}\right) \times \frac{\partial}{\partial u_{b}}+\left(\mathrm{d} y_{s_{e}}+u_{s_{e}} \mathrm{~d} x_{s_{e}}\right) \times \frac{\partial}{\partial u_{s_{e}}}-\left(\mathrm{d} y_{s_{o}}+u_{s_{o}} \mathrm{~d} x_{s_{o}}\right) \times \frac{\partial}{\partial u_{s_{o}}}
$$

The semispray with supergraph bundle coordinate is given by 


$$
\varepsilon=\frac{\partial}{\partial x_{b}}+\frac{\partial}{\partial x_{s_{e}}}-\frac{\partial}{\partial x_{s_{o}}}+u_{b} \frac{\partial}{\partial y_{b}}+u_{s_{e}} \frac{\partial}{\partial y_{s_{e}}}-u_{s_{o}} \frac{\partial}{\partial y_{s_{o}}}+\varepsilon_{i_{b}} \frac{\partial}{\partial u_{b}}+\varepsilon_{s_{e}} \frac{\partial}{\partial u_{s_{e}}}-\varepsilon_{s_{o}} \frac{\partial}{\partial u_{s_{o}}}
$$

If we calculate the $J$-directional derivative $J(\varepsilon)$ of the semispray $\varepsilon$, we can obtain the Liouville vector field $V$ :

$$
V=J(\varepsilon)=2 u_{b} \frac{\partial}{\partial y_{b}}+2 u_{s_{e}} \frac{\partial}{\partial y_{s_{e}}}-2 u_{s_{o}} \frac{\partial}{\partial y_{s_{o}}} \text {. }
$$

Theorem 2. Euler-Lagrange energy equation in superspace with supergraph bundle coordinate will be obtained as follows:

$$
u_{b} \frac{\partial^{2} L_{b}}{\partial x_{b} \partial u_{b}}+u_{s_{e}} \frac{\partial^{2} L_{s_{e}}}{\partial x_{s_{e}} \partial u_{s_{e}}}+u_{s_{o}} \frac{\partial^{2} L_{s_{o}}}{\partial x_{s_{o}} \partial u_{s_{o}}}-u_{b} \frac{\partial L_{b}}{\partial y_{b}}-u_{s_{e}} \frac{\partial L_{s_{e}}}{\partial y_{s_{e}}}-u_{s_{o}} \frac{\partial L_{s_{o}}}{\partial y_{s_{o}}}=0
$$

Proof. We know that the Euler-Lagrange energy can be obtained by solving the dynamical equation $i_{\varepsilon} \Omega_{L}=\Omega_{L}(\varepsilon)=0$. To solve this dynamic equation with graph bundle coordinates, in the proof of Theorem 1, it is sufficient to make the following arrangement in coordinates:

$$
\left(t, x_{i}, \dot{x}_{i}\right)=\left(t_{b}, t_{s_{e}}, t_{s_{o}}, x_{i_{b}}, x_{i_{s_{e}}}, x_{i_{s_{o}}}, \dot{x}_{i_{b}}, \dot{x}_{i_{s_{e}}}, \dot{x}_{i_{s_{o}}}\right)=(x, y, u)=\left(x_{b}, x_{s_{e}}, x_{s_{o}}, y_{b}, y_{s_{e}}, y_{s_{o}}, u_{b}, u_{s_{e}}, u_{s_{o}}\right)
$$

Since equation (29) will be obtained in the same way as the graph bundle coordinates, a system similar to system (30) is obtained. When system (30) is solved under conditions,

$$
\begin{aligned}
& \dot{\varepsilon}_{i_{b}}=-u_{b}, \\
& \dot{\varepsilon}_{i_{s_{e}}}=-u_{s_{e}}, \\
& \dot{\varepsilon}_{i_{s_{o}}}=-u_{s_{o}},
\end{aligned}
$$

by the linear relation which is similar to the linear relation we presented in the proof of Theorem 1,

$$
-u_{b}(1)-u_{s_{e}}(2)+u_{s_{o}}(3)+(4)+(5)-(6)-u_{b}(7)-u_{s_{e}}(8)+u_{s_{o}}(9)=0
$$

time-dependent Euler-Lagrange energy equation (37) is obtained.

Equation (37) can be written more simply depending on the supergraph bundle coordinates, so that

$$
\begin{aligned}
& u\left(\frac{\partial^{2} L}{\partial x \partial u}\right)-u \frac{\partial L}{\partial y}=0 \\
& u \frac{\partial}{\partial u}\left(\frac{\partial L}{\partial x}\right)-u \frac{\partial L}{\partial y}=0 .
\end{aligned}
$$

Equation (41) represents again the Lagrangian energy equation on the supergraph bundle.

We will give an example to investigate the geometric and physical results of this study.

Example 1. In this example, we will define a logarithmic spiral curve in superspace and obtain the energy equation for a particle moving on it (Figure 4). The reason we prefer the logarithmic spiral curve is the equiangular spiral has a lot longer history in the science of mathematics. For example, a hawk follows a logarithmic spiral as it approaches its prey from the air. Tropical hurricanes take the form of a logarithmic spiral.

The logarithmic spiral is given by $\left(t, a(t) e^{b(t) \theta}\right.$ $\left.\cos \theta, a(t) e^{b(t) \theta} \sin \theta\right)$ in parametric form.

Its parametric representation is given by (here $t$ is time parameter, $r$ is the distance from the origin, and $\theta$ is the angle from the $x$-axis)

$$
\begin{aligned}
& x(\theta)=r(\theta) \cos \theta=a e^{b \theta} \cos \theta, \\
& y(\theta)=r(\theta) \cos \theta=a e^{b \theta} \sin \theta .
\end{aligned}
$$

The coordinate system of the jet bundle structure for the superlogarithmic spiral curve is expressed as 


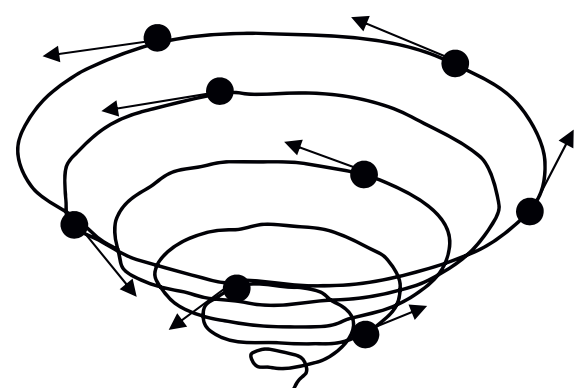

(a)

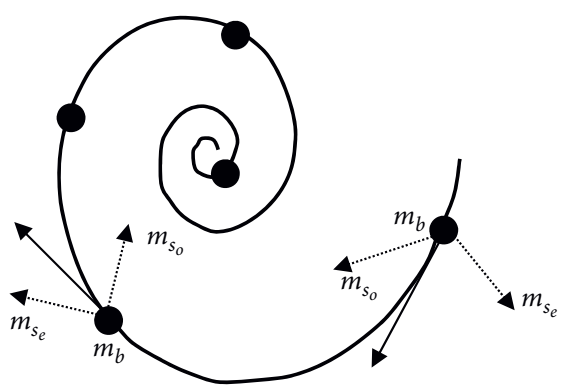

(b)

Figure 4: Motion of a particle on a logarithmic spiral. (a) General view of the movement and (b) the direction of the movement according to the body, soul even, and soul odd parts.

$$
\begin{aligned}
& \left(t, a(t) e^{b(t) \theta} \cos \theta, a(t) e^{b(t) \theta} \sin \theta, a_{t} e^{b(t) \theta} \cos \theta a b_{t} e^{b(t) \theta} \cos \theta, a_{t} e^{b(t) \theta} \sin \theta a b_{t} e^{b(t) \theta} \sin \theta\right) \\
& t_{b}, t_{s_{e}}, t_{s_{o}}, a_{b}(t) e^{b_{b}(t) \theta} \cos \theta, a_{s_{e}}(t) e^{b_{s_{e}}(t) \theta} \cos \theta a_{s_{o}}(t) e^{b_{s_{o}}(t) \theta} \cos \theta, a_{b}(t) e^{b_{b}(t) \theta} \sin \theta, \\
& =\left(\begin{array}{c}
a_{s_{e}}(t) e^{b_{s_{e}}(t) \theta} \sin \theta a_{s_{o}}(t) e^{b_{s_{o}}(t) \theta} \sin \theta, a_{t b} e^{b_{b}(t) \theta} \cos \theta+a_{b} b_{t b} e^{b_{b}(t) \theta} \cos \theta, a_{t s_{e}} e^{b_{s_{e}}(t) \theta} \cos \theta+a_{s_{e}} b_{t s_{e}} e^{b_{s_{e}}(t) \theta} \cos \theta, \\
a_{t s_{o}} e^{b_{s_{o}}(t) \theta} \cos \theta+a_{s_{o}} b_{t s_{o}} e^{b_{s_{o}}(t) \theta} \cos \theta a_{t b} e^{b_{b}(t) \theta} \sin \theta+a_{b} b_{t b} e^{b_{b}(t) \theta} \sin \theta, \\
a_{t s_{e}} e^{b_{s_{e}}(t) \theta} \sin \theta+a_{s_{e}} b_{t s_{e}} e^{b_{s_{e}}(t) \theta} \sin \theta, a_{t s_{o}} e^{b_{s_{o}}(t) \theta} \sin \theta+a_{s_{o}} b_{t s_{o}} e^{b_{s_{o}}(t) \theta} \sin \theta
\end{array}\right) .
\end{aligned}
$$

Firstly, we write the Lagrangian energy equation with these superbundle coordinates. With logarithmic spiral bundle coordinates, if we write and solve equation (33) that we have obtained in Section 3, we obtain Lagrangian energy equations of the particle:

$r_{t_{b}} \frac{\partial^{2} L_{b}}{\partial t_{b} \partial r_{t_{b}}}+r_{t_{s_{e}}} \frac{\partial^{2} L_{s_{e}}}{\partial t_{s_{e}} \partial r_{t_{s_{e}}}}+r_{t_{s_{o}}} \frac{\partial^{2} L_{s_{o}}}{\partial t_{s_{o}} \partial r_{t_{s_{o}}}}-r_{t_{b}} \frac{\partial L_{b}}{\partial r_{b}}-r_{t_{s_{e}}} \frac{\partial L_{s_{e}}}{\partial r_{s_{e}}}-r_{t_{s_{o}}} \frac{\partial L_{s_{o}}}{\partial r_{s_{o}}}=0$.

Here, the Lagrangian energy function is represented as

$$
L=L(r, t)=L_{b}\left(r_{b}, t_{b}\right)+L_{s_{e}}\left(r_{s_{e}}, t_{s_{e}}\right)+L_{s_{o}}\left(r_{s_{o}}, t_{s_{o}}\right) \text {. }
$$

Similar to other spaces, the general equation of Lagrangian energy is the same in superspace even in different motion models.

For the solution of this Lagrangian energy equation, we can take the following assumptions:

$$
\begin{gathered}
\frac{\partial L_{b}}{\partial t_{b}}=\lambda_{b}, \\
\frac{\partial L_{s_{e}}}{\partial t_{s_{o}}}=\lambda_{s_{e}} \\
\frac{\partial L_{s_{o}}}{\partial t_{s_{o}}}=\lambda_{s_{o}} .
\end{gathered}
$$

We reorganize equation (44) with these assumptions:

$$
r_{t_{b}}\left(\frac{\partial \lambda_{b}}{\partial r_{t_{b}}}-\frac{\partial L_{b}}{\partial r_{b}}\right)+r_{t_{s_{e}}}\left(\frac{\partial \lambda_{s_{e}}}{\partial r_{t_{s_{e}}}}-\frac{\partial L_{s_{e}}}{\partial r_{s_{e}}}\right)+r_{t_{s_{o}}}\left(\frac{\partial \lambda_{s_{o}}}{\partial r_{t_{s_{o}}}}-\frac{\partial L_{s_{o}}}{\partial r_{s_{o}}}\right)=0 .
$$

Since the radius cannot be equal to zero,

$$
\begin{gathered}
\frac{\partial \lambda_{b}}{\partial r_{t_{b}}}-\frac{\partial L_{b}}{\partial r_{b}}=0, \\
\frac{\partial \lambda_{s_{e}}}{\partial r_{t_{s_{e}}}}-\frac{\partial L_{s_{e}}}{\partial r_{s_{e}}}=0, \\
\frac{\partial \lambda_{s_{o}}}{\partial r_{t_{s_{o}}}}-\frac{\partial L_{s_{o}}}{\partial r_{s_{o}}}=0 .
\end{gathered}
$$

If these equations are solved, we can obtain the following:

$$
\begin{gathered}
L_{b}=\frac{r_{b} c_{b}+k_{b}}{\ln t_{b}}, \\
L_{s_{e}}=\frac{r_{s_{e}} c_{s_{e}}+k_{s_{e}}}{\ln t_{s_{e}}}, \\
L_{s_{o}}=\frac{r_{s_{o}} c_{s_{o}}+k_{s_{o}}}{\ln t_{s_{o}}},
\end{gathered}
$$

where $c_{b}, c_{s_{e}}, c_{s_{o}}, k_{b}, k_{s_{e}}, k_{s_{o}}$ are constants. Also, the Lagrangian energy function is given by

$$
L=\frac{r_{b}(\theta) c_{b}+k_{b}}{\operatorname{lnt} t_{b}}+\frac{r_{s_{e}}(\theta) c_{s_{e}}+k_{s_{e}}}{\operatorname{lnt}_{s_{e}}}+\frac{r_{s_{o}}(\theta) c_{s_{o}}+k_{s_{o}}}{\operatorname{lnt} t_{s_{o}}} .
$$

We can write this equation for a superpoint briefly as follows: $L=(r . c+k / \operatorname{lnt}),(c, k$ is constant $)$

$$
L=\frac{a(t) e^{b(t)} \cdot c+k}{\ln t}
$$


It is seen that energy depends on radius and time.

Now we write and solve the Lagrangian energy equation with supergraph bundle coordinates.

It is best to determine the turning points of the movement on this curve with graph points because using graph points helps to determine the most appropriate movement according to the increase or decrease in the curvature value of the curve. $a_{0}$ is the starting point and $a_{n}$ is the destination point (Figure 5). This segmentation can be taken with the preferred number of points in accordance with the movement. Figure 5 shows the vertex points through which the moving particle passes and the relationship between the arc length and the length between these points.

In Figure 5, the interpretation of the movement formed by graph points according to the parts is shown. The position of the vertex and edges according to the body, soul odd, and soul even parts for the movement on the logarithmic spiral with supergraph coordinates is shown in more detail in Figure 5. While building this, we used the $\beta$ transformations we defined for the differentiable structure of the graph manifold. At the starting point, we assume that the movement starts from the body part. At the next points, movement will take form between these vertex points, depending on whether the number is an even supernumber or an odd supernumber (that is, it can only have soul even or soul odd part, or it can have body and soul even or body and soul odd part) or every part (from body, soul even, and soul odd parts). The energy value will also be calculated according to the occurrence of the movement.

Graph bundle coordinates according to the parameterization in (54) are given by

$$
\left(x_{b}(\theta), x_{s_{e}}(\theta), x_{s_{o}}(\theta), y_{b}(\theta), y_{s_{e}}(\theta), y_{s_{o}}(\theta), u_{b}(\theta), u_{s_{e}}(\theta), u_{s_{o}}(\theta)\right) \text {. }
$$

Since we will work with the graph space structure, we have said before that we will take the time as a natural coordinating of the physical structure. So, we can write the coordinate structure in more detail as follows:

$$
\begin{aligned}
& \left(t, a(t) e^{b(t) \theta} \cos \theta, a(t) e^{b(t) \theta} \sin \theta, a(t) e^{b(t) \theta} \sin \theta-a(t) e^{b(t) \theta} \cos \theta\right) \\
& =\left(t_{b}, t_{s_{e}}, t_{s_{o}}, a_{b}(t) e^{b_{b}(t) \theta} \cos \theta, a_{s_{e}}(t) e^{b_{s_{e}}(t) \theta} \cos \theta, a_{s_{o}}(t) e^{b_{s_{o}}(t) \theta} \cos \theta, a_{b}(t) e^{b_{b}(t) \theta} \sin \theta,\right) \\
& a_{s_{e}}(t) e^{b_{s_{e}}(t) \theta} \sin \theta a_{s_{o}}(t) e^{b_{s_{o}}(t) \theta} \sin \theta, a_{b} e^{b_{b}(t) \theta} \sin \theta-a_{b}(t) e^{b_{b}(t) \theta} \cos \theta, a_{t s_{e}} e^{b_{s_{e}}(t) \theta} \\
& \sin \theta-a_{s_{e}}(t) e^{b_{s_{e}}(t) \theta} \cos \theta, a_{t s_{o}} e^{b_{s_{o}}(t) \theta} \sin \theta-a_{s_{o}}(t) e^{b_{s_{o}}(t) \theta} \cos \theta .
\end{aligned}
$$

We rewrite equation (37) according to these coordinates and solve it similarly to the solution of the equation we have obtained with the superbundle coordinates:

$$
\frac{1}{\cos \theta}\left[r_{b} \frac{\partial^{2} L_{b}}{\partial r_{b} \partial r_{b}}+r_{s_{e}} \frac{\partial^{2} L_{s_{e}}}{\partial r_{s_{e}} \partial r_{s_{e}}}+r_{s_{o}} \frac{\partial^{2} L_{s_{o}}}{\partial r_{s_{o}} \partial r_{s_{o}}}\right]-(1-\cot \theta)\left[r_{b} \frac{\partial L_{b}}{\partial r_{b}}+r_{s_{e}} \frac{\partial L_{s_{e}}}{\partial r_{s_{e}}}+r_{s_{o}} \frac{\partial L_{s_{o}}}{\partial r_{s_{o}}}\right]=0
$$

When the Lagrangian energy function is calculated from this equation, the following energy equation is obtained:

$$
L=\frac{1}{\varepsilon(\theta)}\left[e^{\varepsilon(\theta) r_{b}(t)} c_{b}+k_{b}+e^{\varepsilon(\theta) r_{s_{e}}(t)} c_{s_{e}}+k_{s_{e}}+e^{\varepsilon(\theta) r_{s_{o}}(t)} c_{s_{o}}+k_{s_{o}}\right] .
$$

Here, $\varepsilon(\theta)=\cos \theta-\cot \theta \operatorname{cosec} \theta$.

We can write this equation for a superpoint briefly as follows:

$$
\begin{aligned}
& L=e^{r(t)} c+k \quad(c, k \text { is constan } t), \\
& L=\frac{1}{\varepsilon(\theta)} e^{\varepsilon(\theta) a(t) e^{b(t)}} c+k .
\end{aligned}
$$

When we think of equations (56) and (51) without the constants, we will see an exponential map formed between these equations. This is a transformation that we interpret as a vector flow in differential geometry. Namely, $X$ is a tangent vector and $\varnothing_{X}$ is a flow of $X$.

$$
\begin{aligned}
& \exp : T_{p} M \longrightarrow M, \\
& \exp (t X)=\varnothing_{X}(t) .
\end{aligned}
$$

The geometrical interpretation of this is that instead of calculating the energy value with superbundle coordinates, when we calculate the energy on the parts of each vertex point and take it as the linear sum of the energy values we find, the energy value we will find as the result of equation (56) and the energy value we will calculate with (50) equation will correspond to the same Lagrangian energy. Since the derivative coordinate corresponds to the edge coordinate, as it physically corresponds to the path between two vertexes for movement, it will be sufficient to calculate the energy formed between each vertex depending on the 


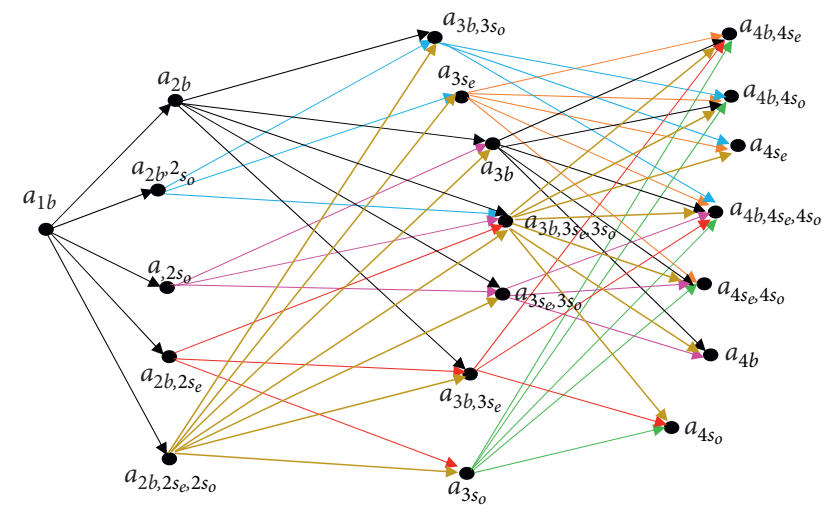

Figure 5: The position of coupling the vertex and edges according to the body, soul odd, and soul even parts for the logarithmic spiral.

edge coordinate on these points. The exponential map states that the energy of the moving particle passing through these vertexes can be calculated with a vector flow corresponding to derivative coordinates between each vertex.

$\varepsilon(\theta)=0$ in equation (56) is a result that affects the physical explanation of the motion. If $\theta=(\pi / 2)$, the orbit is

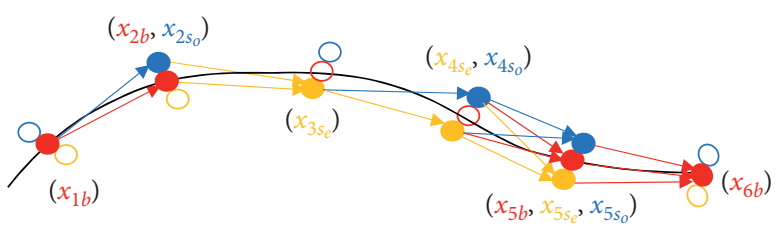

Figure 6: An example of different orientations that can occur between six points for the movement.

circular (if $\theta=(\pi / 2)$, this corresponds to a Fibonacci spiral, which is not the curve we prefer to study in this example). In this case, there is no slipping and deviation in motion; it goes completely linear. If $0 \leq \theta \leq(\pi / 2)$, the rotation will be less for the orbit more closed. If $(\pi / 2) \leq \theta \leq \pi$, since the movement occurs at a great angle, there will be more turns.

For example, as shown in Figure 6, the first point has only the body part, the second point has body and soul even parts, the third point has only soul even part, the fourth point has soul even and soul odd parts, and the fifth point has body, soul even, and soul odd parts. The last point has only the body part like the beginning. Then the Lagrangian energy generated by these six points is calculated by the following equation:

$$
\begin{aligned}
L= & \frac{1}{\varepsilon(\theta)}\left[e^{\varepsilon(\theta) a_{1 b}(t) e^{b_{1 b}(t)}}+\left(e^{\varepsilon(\theta) a_{2 b}(t) e^{b_{2 b}(t)}}+e^{\varepsilon(\theta) a_{2 e_{e}}(t) e^{b_{25 e}(t)}}\right)+\left(e^{\varepsilon(\theta) a_{3 s_{e}}(t) e^{b_{3 s_{e}}(t)}}\right)+\left(e^{\varepsilon(\theta) a_{4 s_{e}}(t) e^{b_{4 s_{e}}(t)}}-e^{\varepsilon(\theta) a_{4 s_{o}}(t) e^{b_{4 s_{o}}(t)}}\right)\right. \\
& \left.+\left(e^{\varepsilon(\theta) a_{5 b}(t) e^{b_{5 b}(t)}}+e^{\varepsilon(\theta) a_{5 s_{e}}(t) e^{b_{5 s_{e}}(t)}}-e^{\varepsilon(\theta) a_{5 s_{o}}(t) e^{b_{5 s_{o}}(t)}}\right)+e^{\varepsilon(\theta) a_{6 b}(t) e^{b_{6 b}(t)}}\right] c+k
\end{aligned}
$$

\section{Conclusion and Discussion}

In this study, firstly Lagrangian energy equations in superspace have been obtained by defining necessary geometric structures. It is advantageous to use bundle structures for proofs in the presence of time-dependent Lagrangian energy because the solution of mechanical systems is more convenient with bundle structures. In this study, the bundle structure was established in the superspace, and the timedependent energy equation was calculated with these superbundle coordinates. On the other hand, graph theory is the most suitable method for planning a movement that completes a path as soon as possible. For this reason, we formed the graph manifold structure in superspace and formed a supergraph bundle with supergraph manifolds. We have figured thirty-one topological maps that constitute the differential structure of the graph manifold. It is known that supercoordinates consist of body, soul even, and soul odd parts. We adapted this coordinate structure to graph theory and examined it. In addition, we proved the jet bundle required for time-dependent mechanical systems and the derivative coordinate with graphs and interpreted geometrically. Thus, we have obtained the time-dependent Lagrangian energy equation with the supergraph bundle. We compared the energy equation obtained with the superjet bundle with the energy equation obtained with the supergraph bundle. In addition, we show the application of our study on an example and give the physical explication. We showed the vertex and edges on the figure and showed how to write an energy equation with these coordinates.

The following conclusions can be drawn from this study:

(1) For Lagrangian equations obtained by using jet bundles in superspace, the negative definition in coordinates comes from odd parity. Although all obtained geometric structures for superspace contain this negative term, the resulting Lagrange energy equation is independent of this term. This is an interesting result because the negative term is expected to affect the result due to odd parity. But when working with supergraph bundles, odd parity affects the direction. The negative term is not used in the equations of geometric structures. It is explained by orientation on graph.

(2) The general form of the Lagrange energy equation is shown by equation (6). It can be expressed as $\left(\partial^{2} L / \partial t \partial \dot{q}_{i}\right)-\left(\partial L / \partial q_{i}\right)=0$.

Lagrangian energy equation calculated in superspace is shown in (33). This equation can be written for shortness as $\left(\partial^{2} L / \partial t \partial \dot{x}_{i}\right)-\left(\partial L / \partial x_{i}\right)=0\left(\right.$ if $\left.\dot{x}_{i} \neq 0\right)$.

Lagrangian energy equation calculated with supergraph bundle is shown in (37). This equation can be written as $(\partial / \partial u)(\partial L / \partial x)-(\partial L / \partial y)=0$ (if $u \neq 0)$

In our previous work [11], we showed that the Lagrangian energy equation obtained in complex space 
is similar to its general form in Euclid space. In this study, it is seen that the Lagrangian energy equations obtained in superspace are also similar to the general equation. The energy equation in superspace can be projected by equations in Euclidean space or even complex space. The given example supports this subject.

(3) When working with the supergraph bundle, the derivative coordinate corresponds to the edge coordinate, making it easier to calculate the obtained energy equation. Equation (58) is an example of this. This can be seen in the given example. Supergraph manifolds obtained by proving the differential structure will be beneficial for the studies. This study is informative of the combination of graph theory with differential geometry.

(4) Here, it is possible to associate the movement on the curve with the vertexes by using edges to calculate the arc length. $\left(\Delta a_{2} / \Delta a_{1}\right)=\cdots=\left(\Delta a_{i} / \Delta a_{i-1}\right)=s$ (arc length), $\Delta a_{i}=a_{i}-a_{i-1}$.

If $a_{i}$ and $a_{i-1}$ are vertexes, the length $\Delta a_{i}=e_{i}$ corresponds to an edge.

(5) In this article, while the physical interpretation of Lagrangian equations is made, the effect of the parts of coordinates in superspace on this motion is shown. This matter is shown in the figures. This study could lead to further research. One of the interesting conclusions of this study is if the moving object continues to move without stopping at a certain speed, the energy becomes stable after a certain time. This is compatible with the nature of Lagrangian energy.

(6) As a result of given examples, we can see that Lagrangian energy value increases and approaches a fixed value based on time. Obviously, the stabilization of superenergy is analogous to the speed of light. In addition, as shown in the example, it is useful to use graph points to determine the most suitable path when calculating the energy of a moving particle. Thus, we can evaluate the energy for each vertex separately and examine even an infinite motion as if it is finite.

\section{Data Availability}

Data analyzed in this study were a re-analysis of existing data, which are openly available at locations cited in the reference section. Further documentation about data processing is available at [Journal of Mathematic] at doi.org/ $10.1155 / 2021 / 5528123$.

\section{Additional Points}

Summary. Our study focuses on obtaining the Lagrangian energy equations in superspace with superjet bundle coordinates. Therefore, the solution method is completely different. In addition, it is more advantageous to work with bundles in mechanics. In our study, these differences are interpreted with given examples. In the examples, we will use the graph theory to determine the optimal motion, velocity, and energy of the particle, due to graph points. These assumptions will be useful for similar studies (https://1drv.ms/ b/s!Ah4SvAdAlNDmggMghTVu-a7Pk-9C).

\section{Conflicts of Interest}

The authors declare that they have no conflicts of interest.

\section{References}

[1] Y. Norimasa, H. Tamayuki, and S. Takafumu, "Interactive control of planar class a bezier curves using logarithmic curvature graphs," Computer- Aided Design and Applications, vol. 5, no. 1-4, pp. 121-130, 2008.

[2] C. Miles, S. Greydanus, and S. Hoyer, Lagrangian Neural Networks, 2020, https://arxiv.org/abs/2003.04630.

[3] İ.N. Cangül, Graph Theory 1, Dora Yayıncllik, Istanbul, Turkey, 2017.

[4] İ.N. Cangül and S. Delen, "A new graph invariant," Turkish Journal of Analysis and Number Theory, vol. 6, no. 1, pp. 30-33, 2018.

[5] T. Berry and S. Schluchter, "Applications of topological graph theory to 2-manifold learning," 2018, https://arxiv.org/abs/ 1809.00050 .

[6] E. Bolker, V. Guillemin, and T. Holm, "How is a graph like a manifold," 2002, https://arxiv.org/abs/math/0206103.

[7] S. Buyalo and P. Svetlov, "Topological and geometric properties of graph manifolds," St. Petersburg Mathematical Journal, vol. 16, no. 2, pp. 297-340, 2005.

[8] F. Waldhausen, "Gruppen mit Zentrum und 3-dimensionale Mannigfaltigkeiten,” Topology, vol. 6, no. 4, pp. 505-517, 1967.

[9] W. Imrich, T. Pisanski, and J. Żerovnik, "Recognizing cartesian graph bundles," Discrete Mathematics, vol. 167-168, pp. 393-403, 1997.

[10] C. Aycan, "The lifts of Euler-Lagrange and Hamiltonian equations on the extended jet bundles," Ph.D. thesis, Osmangazi University, Eskisehir, Turkey, 2003.

[11] C. Aycan and S. Dagli, "Improving Lagrangian energy equation on the Kahler jet bundles," International Journal of Geometric Methods in Modern Physics, vol. 10, no. 7, Article ID 1350026, 2013.

[12] S. Dagli, "The jet structure and mechanical systems on Minkowski 4-Space," Ph.D. thesis, Pamukkale University, Denizli, Turkey, 2012.

[13] M. De Leon, J. M. Solano, and j.c. Marrero, "Singular Lagrangian systems on jet bundles," Fortschritte der Physik, vol. 50, no. 2, pp. 105-169, 2001.

[14] M. Crampin, "Lagrangian submanifolds and the EulerLagrange equations in higher-order mechanics," Letters in Mathematical Physics, vol. 19, no. 1, pp. 53-58, 1990.

[15] M. De Leon, D. Chinea, and J. C. Marrero, "The constraint algorithm for time dependent Lagrangians," Publ. Univ. La Laguna. Serie Informes, vol. 32, pp. 13-29, 1991.

[16] G. Sardanashvily, "Classical and quantum mechanics with time-dependent parameters," Journal of Mathematical Physics, vol. 41, no. 8, pp. 5245-5255, 2000.

[17] M. De Leon and P. R. Rodrigues, Generalized Classical Mechanics and Field Theory, vol. 112, North- Hol. Math. St., Elsevier Sc. Pub., Amsterdam, Netherlands, 1991.

[18] A. Jadczyk and K. Pilch, "Superspaces and supersymmetries," Communications in Mathematical Physics, vol. 78, no. 3, pp. 373-390, 1981. 
[19] C. Bartocci and U. Bruzzo, The Geometry of Super Manifolds, Springer, Dordrecht, Netherlands, 1991.

[20] A. J. Bruce, On Curves and Jets of Curves on Supermanifolds, Cornell University Library, Ithaca, NY, USA, 2014, https:// arxiv.org/abs/1401.5267.

[21] T. D. Parsons and T. Pisanski, "Vector representations of graphs," Discrete Mathematics, vol. 78, no. 1-2, pp. 143-154, 1989.

[22] G. Sardanashvily, Lectures on Supergeometry, Cornell University Library, Ithaca, NY, USA, 2009, https://arxiv.org/abs/ 0910.0092 .

[23] A. Rogers, Supermanifolds, Theory and Applications, World Scientific Pub., Singapore, 2007.

[24] G. Sardanashvily, "Supermetrics on supermanifolds," International Journal of Geometric Methods in Modern Physics, vol. 05 , no. 02 , pp. 271-286, 2008. 